\title{
Wilms tumour
}

Filippo Spreafico $\mathbb{1}^{1 凶}{ }^{凶}$, Conrad V. Fernandez $\mathbb{1}^{2}$, Jesper Brok ${ }^{3}$, Kayo Nakata ${ }^{4}$, Gordan Vujanic (1) ${ }^{5}$, James I. Geller ${ }^{6}$, Manfred Gessler (1) ${ }^{7}$, Mariana Maschietto (1) ${ }^{8}$, Sam Behjati ${ }^{9,10,11}$, Angela Polanco ${ }^{12}$, Vivian Paintsil $\mathbb{1}^{13}$, Sandra Luna-Fineman (1) ${ }^{14}$ and Kathy Pritchard-Jones (1D ${ }^{15}$

Abstract / Wilms tumour (WT) is a childhood embryonal tumour that is paradigmatic of the intersection between disrupted organogenesis and tumorigenesis. Many WT genes play a critical (non-redundant) role in early nephrogenesis. Improving patient outcomes requires advances in understanding and targeting of the multiple genes and cellular control pathways now identified as active in WT development. Decades of clinical and basic research have helped to gradually optimize clinical care. Curative therapy is achievable in $90 \%$ of affected children, even those with disseminated disease, yet survival disparities within and between countries exist and deserve commitment to change. Updated epidemiological studies have also provided novel insights into global incidence variations. Introduction of biology-driven approaches to risk stratification and new drug development has been slower in WT than in other childhood tumours. Current prognostic classification for children with WT is grounded in clinical and pathological findings and in dedicated protocols on molecular alterations. Treatment includes conventional cytotoxic chemotherapy and surgery, and radiation therapy in some cases. Advanced imaging to capture tumour composition, optimizing irradiation techniques to reduce target volumes, and evaluation of newer surgical procedures are key areas for future research.

Nephron-sparing surgery An operation to remove a kidney tumour by removing only part of the surrounding normal renal parenchyma. 凶e-mail: filippo.spreafico@ istitutotumori.mi.it

https://doi.org/10.1038/ s41572-021-00308-8
Wilms tumour (WT) is the most common renal tumour of infants and young children ${ }^{1,2}$. WT is intimately linked to early nephrogenesis, which it resembles morphologically ${ }^{3}$ and transcriptionally ${ }^{4,5}$. WT may occur sporadically or in the context of bilateral tumours, multifocal disease and specified genetic predisposition syndromes that frequently include either genitourinary malformation or overgrowth ${ }^{3}$. Beyond genetic predisposition, external causative factors for WT are not yet defined. The molecular drivers frequently involve blockade of genetic pathways that guide normal embryogenesis of the genitourinary tract but are not restricted to these. Indeed, the cancer genes that underpin WT are diverse and surprisingly involve $\sim 40$ genes.

The implementation of international co-operative group trials and studies across North America, Australia, New Zealand, Europe and Brazil has contributed significantly to improving outcomes ${ }^{6-8}$. Two international multidisciplinary cooperative consortia - the Children's Oncology Group (COG) Renal Tumour Committee, previously known as the National Wilms Tumour Study Group (NWTSG), and the International Society of Paediatric Oncology (SIOP) Renal Tumour Study Group (RTSG) - have conducted large multicentre studies since 1969 and 1971, respectively, which have defined the current diagnostic and therapeutic approach to patients with WT (FIG. 1). These groups continue research to optimize disease and patient risk classification and treatment strategies ${ }^{9-11}$.

In the COG, WTs are treated with primary resection (if possible), followed by risk-adapted adjuvant therapy, whereas in the context of SIOP cooperation, neoadjuvant chemotherapy followed by resection and adjuvant therapy is the preferred treatment approach. Regardless of the initial approach, the overall survival of children with WT is remarkable with rates of $>90 \%$. Such satisfying survival rates have been achieved at the same time as fine-tuning treatment by adopting well-studied prognostic factors, leading to a two-drug regimen (vincristine and actinomycin D) prescribed in nearly two-thirds of affected children ${ }^{7,10}$. Notably, striking survival disparities still exist within countries ${ }^{12}$ and between different parts of the world, which remain to be addressed ${ }^{13,14}$. However, $20 \%$ of patients relapse after first-line therapy and up to $25 \%$ of survivors report severe late morbidity of treatment ${ }^{15,16}$. Addressing the long-term effect of radical nephrectomy on renal function and cardiovascular function will probably drive more attention on expanding the role of nephron-sparing surgery (NSS) ${ }^{17}$.

Molecular studies are expanding the landscape of cancer genes implicated in WT beyond exclusive roles in nephrogenesis ${ }^{3}$. The use of next-generation 


\author{
Author addresses \\ ${ }^{1}$ Department of Medical Oncology and Hematology, Paediatric Oncology Unit, \\ Fondazione IRCCS Istituto Nazionale dei Tumori, Milan, Italy. \\ ${ }^{2}$ Department of Paediatrics, IWK Health, Dalhousie University, Halifax, \\ Nova Scotia, Canada. \\ ${ }^{3}$ Department of Paediatric Haematology and Oncology, Rigshospitalet, Copenhagen, \\ Denmark. \\ ${ }^{4}$ Cancer Control Center, Osaka International Cancer Institute, Osaka, Japan. \\ ${ }^{5}$ Department of Pathology, Sidra Medicine, Doha, Qatar. \\ ${ }^{6}$ Cincinnati Children's Hospital Medical Center, University of Cincinnati, Cincinnati, \\ Cincinnati, OH, USA. \\ 'Theodor-Boveri-Institute, Developmental Biochemistry, and Comprehensive Cancer \\ Center Mainfranken, University of Wuerzburg, Wuerzburg, Germany. \\ ${ }^{8}$ Research Center, Boldrini Children's Hospital, Genetics and Molecular Biology, Institute \\ of Biology, State University of Campinas, Campinas, SP, Brazil. \\ ${ }^{9}$ Wellcome Sanger Institute, Hinxton, UK. \\ ${ }^{10} \mathrm{Cambridge}$ University Hospitals NHS Foundation Trust, Cambridge, UK. \\ ${ }^{11}$ Department of Paediatrics, University of Cambridge, Cambridge, UK. \\ ${ }^{12}$ National Cancer Research Institute Children's Group Consumer Representative, \\ London, UK. \\ ${ }^{13}$ Department of Child Health, School of Medicine and Dentistry, Kwame Nkrumah \\ University of Science and Technology, Kumasi, Ghana. \\ ${ }^{14}$ Division of Hematology, Oncology and Bone Marrow Transplantation, Department \\ of Paediatrics, University of Colorado, Aurora, CO, USA. \\ ${ }^{15}$ Developmental Biology and Cancer Research and Teaching Department, UCL Great \\ Ormond Street Institute of Child Health, University College London, London, UK.
}

Nephrogenic rests

Abnormally persistent foci of embryonal cells regarded as precursor lesions of Wilms tumour. Rests are subdivided into two main types: perilobar, confined to the periphery of the renal lobe, and intralobar found anywhere within the renal lobe.

Overgrowth syndromes A heterogeneous group of disorders in which the main characteristic is that weight, height or head circumference is two to three standard deviations above the mean for sex and age. The different presentations are dependent on the developmental pathways and organ systems affected.

\section{Aniridia}

A rare condition characterized

by a partial or complete

absence of the iris of the eye.

Nephrotic syndrome

A rare clinical disorder defined by massive proteinuria

(>40 mg/m² per hour)

responsible for

hypoalbuminaemia $(<25 \mathrm{~g} / \mathrm{l})$, with resulting hyperlipidaemia, oedema, and various complications. integrative genomic and epigenetic tumour analysis has provided important insights into WT biology. Comparisons of the regulation of progenitor cells in the fetal kidney with the disrupted regulation of their counterparts in WT should provide further insights into tumour formation ${ }^{18}$. Targeting WT tumour genes with a non-redundant role in nephrogenesis and targeting the fetal renal transcriptome warrant further therapeutic exploration. Interventions that could prevent the evolution of nephrogenic rests to malignant WT could transform therapy in this setting and could even lead to preventive strategies in children known to be at high risk of developing WT.

This Primer describes our current understanding of WT epidemiology, disease susceptibility and mechanisms, as well as elements of clinical care, including diagnostics and risk-stratified treatment of newly diagnosed disease. In addition, we also outline potential opportunities to further translate new biological insights into improved clinical outcomes. We discuss how the widespread implementation of standardized diagnostics and treatments for as many children as possible, regardless of socioeconomic status or geographical region of origin, may propel further clinical advances.

\section{Epidemiology \\ Global disease burden}

Malignant renal tumours comprise $5 \%$ of all cancers occurring before the age of 15 years $^{19}$. Every year $\sim 14,000$ children (0-14 years of age) are diagnosed worldwide, and 5,000 children die from these diseases, with regional variation in mortality ${ }^{20}$ (FIG. 2). The incidence of childhood renal tumours is not associated with economic status, but mortality is higher in low-income areas than high-income areas ( 0.5 per million in high-income areas versus 7.5 per million in low-income areas).
WT is the most common renal tumour in children and studies have found variation in incidence between regions or ethnicities ${ }^{2,21}$ (FIG. 3). The annual incidence of WT in East Asia is lower than in North America or Europe (4.3 per million versus 8-9 per million) ${ }^{2}$. In the USA, children with African-American ancestry have the highest incidence (9.7 per million) whilst those with Asian-Pacific Islander ancestry have the lowest (3.7 per million) ${ }^{2}$. However, owing to the lack of population-based childhood cancer registries in resource-constrained regions, or because of the low quality of the data (that is, not all cancers are reported or not all children are reported), the estimation of global incidence has been difficult ${ }^{14,22,23}$. In addition, 50\% of patients from areas with less resources have metastases at diagnosis ${ }^{24}$.

Up to $17 \%$ of WT occur as part of a recognizable malformation syndrome ${ }^{25}, 10 \%$ of which are associated with known WT predisposition ${ }^{26}$ (TABLE 1). Overgrowth syndromes, in particular BeckwithWiedemann syndrome, carry $\sim 5 \%$ risk of developing $\mathrm{WT}$, ranging from $0.2 \%$ to $24 \%$ according to the underlying genetic cause $\mathrm{e}^{27-29}$. Syndromes involving genitourinary anomalies combined with aniridia and variable intellectual disability, or with nephrotic syndrome, are associated with mutations of the gene WT1 on chromosome $11 \mathrm{p} 13$ and these patients have a greatly increased risk of developing $\mathrm{WT}^{3,30,31}$.

No temporal trends in the incidence of WT were observed within the period 1996-2010 (REF. ${ }^{2}$ ), suggesting that environmental factors play a marginal role in WT aetiology. Nevertheless, modifiable risk factors for WT are not well understood.

\section{Influence of sex and age}

WT is one of the few childhood cancers that is more common $(\sim 10 \%)$ in girls than in boys ${ }^{19}$. The age-specific incidence of WT peaks at 1 year of age in boys at 17.9 per million person-years. However, in girls, a similar peak remains almost constant at 1,2 and 3 years of age, with the respective incidences of $17.8,18.0$ and 18.1 per million person-years (FIG. 4).

WT often presents as a solitary lesion, but $\sim 7 \%$ are reported to be multifocal and 5-9\% bilateral ${ }^{1,2,32}$. Unilateral tumours occur at a slightly older age than bilateral ones (FIG. 4). The age distribution at diagnosis varies by region and ethnicity, with affected individuals in East Asia being younger at diagnosis than those in the rest of the world, and this observation may be mainly due to earlier onset of the disease $e^{2,21,33}$ (FIG. 4). As one possible reason of the variation in age at onset, somatic tumour genetic analysis has shown a lower frequency of tumours with H19-IGF2 loss of imprinting among Japanese patients with WT than in Caucasian populations ${ }^{34}$. H19-IGF2 loss of imprinting-driven WTs are associated with overgrowth syndromes and with perilobar nephrogenic rests; both these features are more common in Caucasian children with older age at diagnosis than in Japanese children (median age at diagnosis was 39 months in UK patients with WT versus 28 months in a similar Japanese patient cohort) ${ }^{33-35}$. The observation of the incidence peak in infancy and 
WAGR syndrome

A rare contiguous gene deletion syndrome (Wilms tumour (WT), aniridia, genitourinary anomalies, and range of developmental delays associated with a $45-60 \%$ risk of developing WT.

Hypospadias

An anatomical congenital malformation of the male external genitalia,

characterized by abnormal development of the urethral fold and the ventral foreskin of the penis that causes abnormal positioning of the urethral opening

Cryptorchidism

The absence of at least one testicle from the scrotum.

Denys-Drash syndrome A rare condition caused by mutations in the

tumour-suppressor gene WT1 characterized by a triad of disorders: ambiguous genitalia nephrotic syndrome leading to end-stage renal disease, and Wilms tumour. the lower total incidence in East Asian populations is consistent with genetic factors primarily driving WT. Studies with large samples from many countries and different ethnic groups will be needed to validate the likelihood that the genetic heterogeneity of WT explains this variation in clinical features by ethnicity.

\section{Mechanisms/pathophysiology}

WT is an embryonal malignancy thought to arise through abortive or disrupted development ${ }^{36}$. During kidney embryogenesis, intermediate mesoderm differentiates into metanephric mesenchyme, which condenses around the branching ureteric bud structures. This metanephric mesenchyme undergoes a mesenchymal to epithelial transformation to form renal vesicles, which expand and give rise to the majority of cell types of the functional kidney ${ }^{37}$. In WT, this process can be disrupted at different levels, leading to variable mixtures of blastemal, epithelial and stromal cells that may even exhibit myogenic differentiation. Histology is partly shaped by the underlying genetic defects but may also reflect the timing of divergence from normal nephrogenesis (FIG. 5).

Our understanding of the genetic causes of WT has long been limited to mutations of WT1, CTNNB1 and WTX as well as loss of H19-IGF2 imprinting, but these alterations only explain a subset of cases ${ }^{38}$. Additional features such as allele loss on chromosomes $1 \mathrm{p}$ and $16 \mathrm{q}$ or gain of 1q may underpin aggressive clinical behaviour in some cases, but do not provide mechanistic insights into tumour development or therapeutic targets ${ }^{39-41}$.
Next-generation sequencing analyses have unveiled many additional drivers, mostly chromatin-modifying and transcription factors as well as microRNA (miRNA) processing genes, many of which are involved in normal renal development ${ }^{42-44}$ (TABLE 2; BOX 1). A surprisingly large fraction of WT (up to 17\%) occur in the context of genetic malformation syndromes associated with tumour predisposition ${ }^{25}$ (TABLE 1). The paradigms are WAGR syndrome and Beckwith-Wiedemann syndrome, which led to the understanding that defects in the tumour suppressor gene WT1 and loss of H19-IGF2 imprinting predisposes to WT.

\section{WT1, CTNNB1 and stromal WT}

WT1 was originally identified through homozygous deletions in $\mathrm{WT}^{45,46}$. Nevertheless, the functions of this zinc finger protein are more complex - germline inactivation leads to male genitourinary anomalies, such as hypospadias, cryptorchidism, through haploinsufficiency and is associated with an increased risk of developing WT $(>50 \%)^{47}$. Additionally, dominant-negative mutations, especially of the zinc finger domains that abrogate DNA binding, lead to Denys-Drash syndrome with intersex and renal failure due to diffuse mesangial sclerosis and the risk of developing WT increased $>90 \%{ }^{48}$. Of note, Frasier syndrome, in which intronic mutations alter the balance of WT1 splice isoforms rather than altering the WT1 protein amino acid sequence, includes different forms of intersex and renal failure, and carries a risk of gonadoblastoma in streak gonads rather than $\mathrm{WT}^{49,50}$.

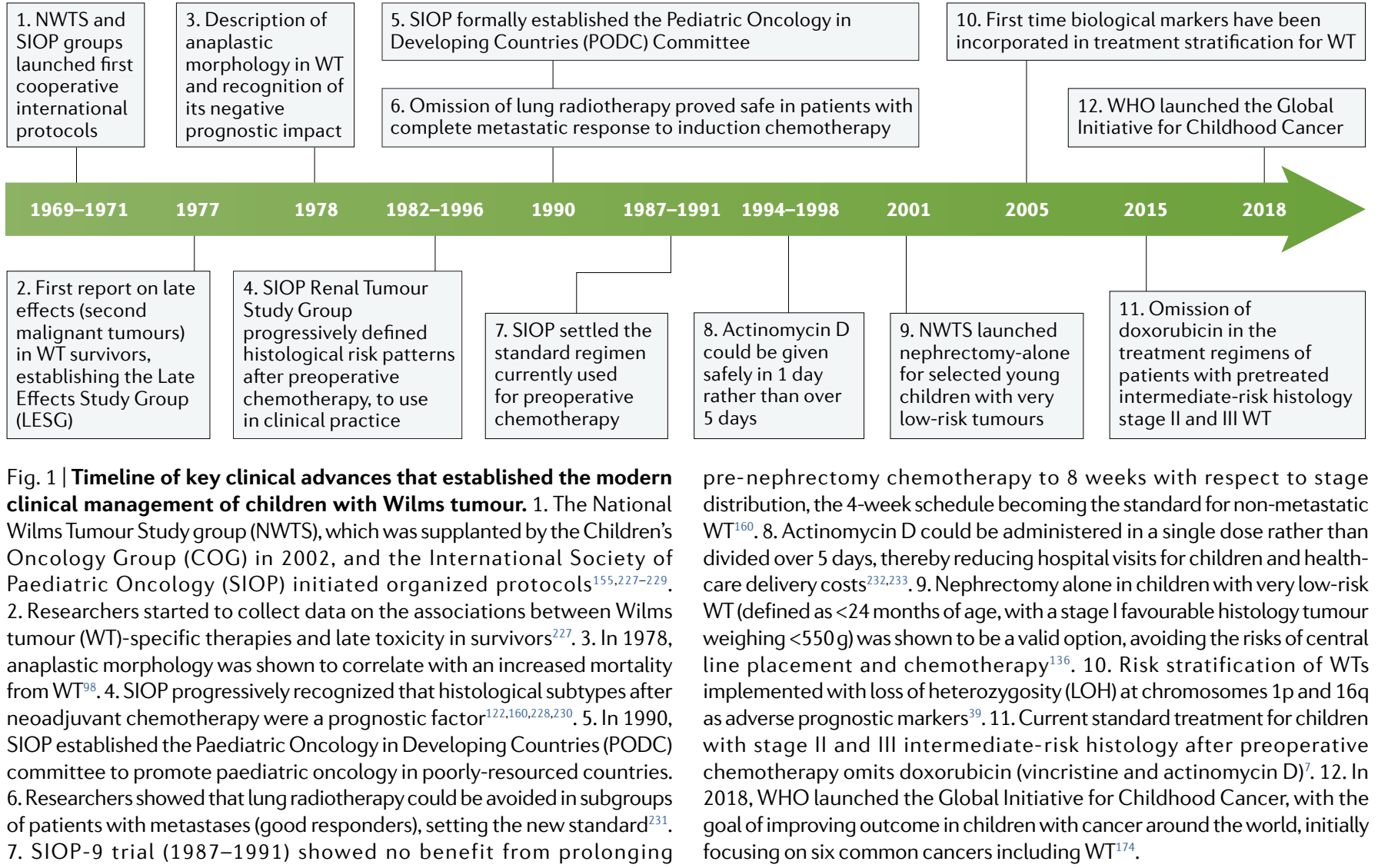




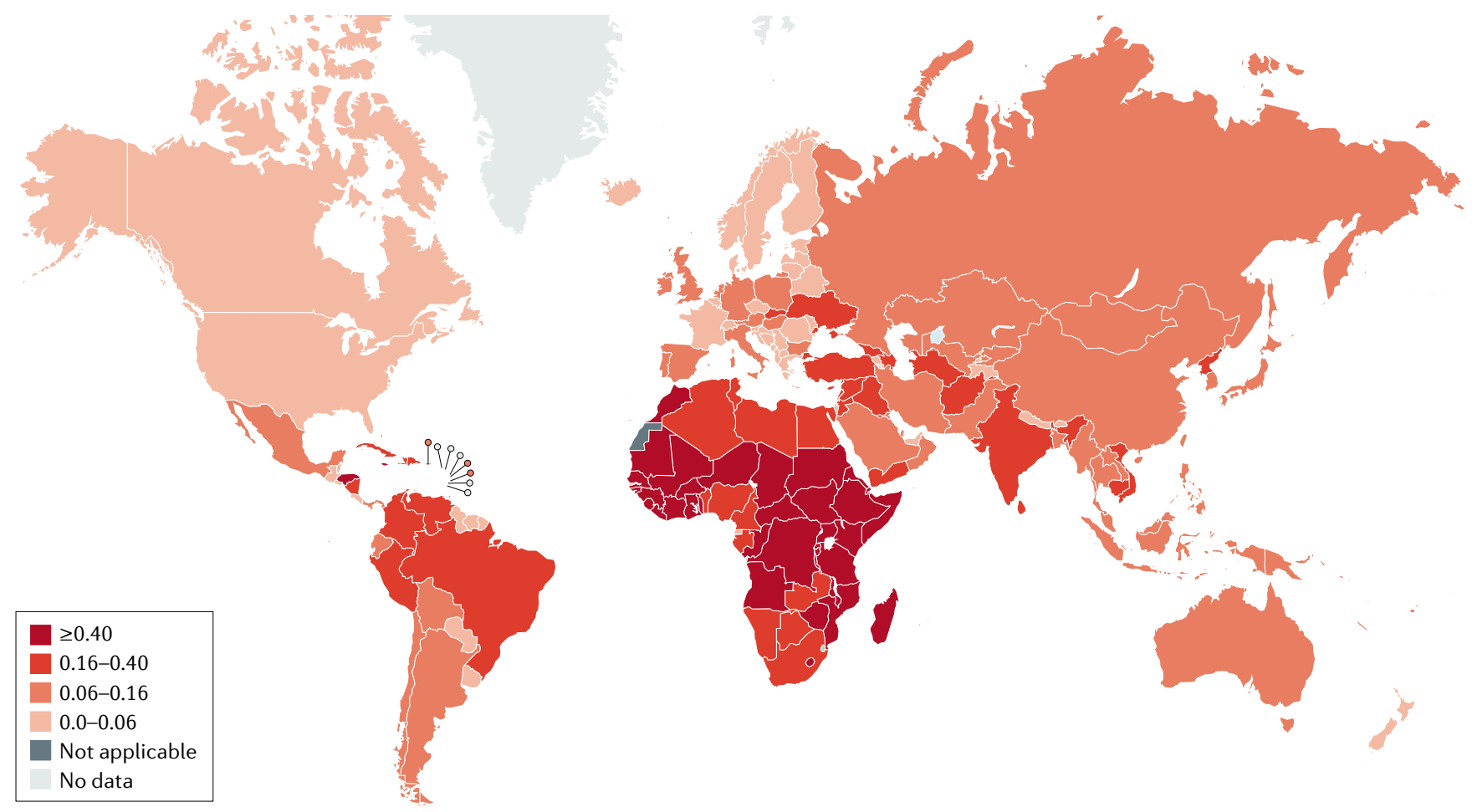

Fig. 2 | The estimated mortality for kidney cancers according to geographical area. Estimated age-standardized mortality rates in 2020 for kidney cancers in children aged $0-14$ years in the world ${ }^{234}$. Reprinted from GLOBOCAN 2020, International Agency for Research on Cancer, Estimated age-standardized mortality rates (world) in 2020, kidney, both sexes, ages 0-14, copyright 2020 (REF. ${ }^{234}$ ).

Mutations in $W T 1$ are often paired with frequent alterations of CTNNB1, which lead to constitutive Wnt signalling ${ }^{51}$. In most cases, point mutations or deletions are observed in the phosphodegron motif in exon 3 , leading to $\beta$-catenin stabilization and nuclear accumulation, where it acts as co-activator with the TCF-LEF transcription factors. These tumours usually exhibit stromal-predominant histology, decreased response to preoperative chemotherapy and represent up to $15 \%$ of cases in Caucasian populations ${ }^{52}$. Notably, although the incidence of WT in Japanese children is only $50 \%$ of that found in Caucasian children, an increased rate of WT1 mutations (81\%) is observed in bilateral WTs, which points to differences in genetic constitutions ${ }^{53}$. WT1-driven stromal tumours occur at a median age of 22 months and are characterized by the presence of intralobar nephrogenic rests as presumed precursor lesions. WTX may likewise facilitate Wnt signalling as it is part of the $\beta$-catenin degradation complex. Mutations or loss of expression of WTX are observed in up to $30 \%$ across histological subtypes, but with intratumoural heterogeneity, suggestive of a late event ${ }^{54}$.

\section{H19-IGF2 imprinting}

Frasier syndrome

A rare autosomal recessive disorder that presents with male pseudohermaphroditism with gonadal dysgenesis, renal failure in early adulthood and increased risk of developing gonadoblastoma. through copy-neutral loss of heterozygosity with invariant loss of the maternal allele or loss of imprinting with epigenetic changes on the maternal allele. The net outcome being hypermethylation of the imprinting centre IC1 with elevated expression of the neighbouring growth factor gene IGF2, among others. With $\sim 70 \%$ incidence
Chromosome 11 p15.5 is frequently altered in WT of such alterations, overexpression of IGF2 is the most frequent change in $\mathrm{WT}^{43,44,52}$. However, tumour initiation may require additional events as loss of imprinting on its own in Beckwith-Wiedemann syndrome only modestly increases WT risk ${ }^{27}$.

\section{microRNA biogenesis mutations}

An unexpected addition to WT driver genes is miRNA processing genes. miRNA biogenesis covers a stepwise maturation process from pri-miRNA via pre-miRNA to mature miRNA. Mutations in WT primarily affect the so-called microprocessor genes DROSHA and DGCR8, which are involved in pri-miRNA processing ${ }^{43,44,55,56}$. Heterozygous DROSHA mutations tend to inactivate the catalytic core of one of the two RNAse III domains that processes pri-miRNA molecules. DGCR 8 mutations affect a single amino acid (E518K) in one of the double-stranded RNA binding domains and the mutations occur homozygously or with monoallelic expression of the mutant. The net result is a reduced and unbalanced miRNA processing. DGCR8 mutations have been observed predominantly in girls, which remains to be explained.

DICER 1, which encodes the second RNAse III type enzyme, is rarely mutated in WT, but predisposes to pleuropulmonary blastoma and is implicated in the very rare entity, anaplastic sarcoma of the kidney ${ }^{57,58}$. The catalytic centre is often mutated on the single functional allele, leading to a partial processing defect with a deficiency in miRNA-5p and largely unaffected miRNA-3p levels.

Studies have found further mutations in XPO5 (encoding exportin 5), the DICER1 cofactor TARBP2 
and downstream let-7 miRNA modulators such as $D I S 3 L 2$ or $L I N 28 B$ at a lower frequency in the $1 \%$ range but the mechanistic details are as yet unclear. Nevertheless, almost all steps of miRNA biogenesis can be critically altered to drive WT formation and several of these mutations are rather specific to WT. The fact that most mutations do not fully abrogate miRNA production implies that specific miRNA subsets are important to control deviation from regular developmental progression or cell proliferation and survival.

\section{MYCN and transcriptional control}

Alterations in MYCN may contribute to WT biology in several ways. Elevated expression levels have been described, especially in relapsing and fatal cases. Furthermore, studies have identified specific $\mathrm{P} 44 \mathrm{~L}$ point mutations or copy number gains with one or a few additional copies ${ }^{44,55,59}$. Proline 44 is located immediately upstream of the conserved MYC box I that interacts with AURKB, FBXW7 (REF. ${ }^{60}$ ) and GSK3 to control N-Myc stability. Stabilization occurs through dephosphorylation at threonine- 58 by the phosphatase EYA1, which is recruited to the nucleus by the transcription factor SIX2 (REF $\left.{ }^{61}\right)$. This process provides a direct link to the paralogous genes SIX1 and SIX2 that control early kidney development. SIX1 and SIX2 can be found as drivers of blastemal-predominant WT if their DNA binding domain becomes subtly altered by stereotypic Q177R mutations ${ }^{44}$.

Intriguingly, several MYC-interacting protein complexes can be targets of mutations in WT. The obligate heterodimerization partner MAX can exhibit R60Q mutations in the helix-loop-helix domain to alter its transcriptional potency. N-Myc exerts its effects on transcriptional control through a multitude of interactions with the core transcriptional machinery to regulate polymerase pausing. The PAF1 transcription complex is one of the critical interactors in this respect and several of its components such as CDC73, MLLT1-ENL and CTR9 have been shown to be mutated in familial and sporadic cases of $\mathrm{WT}^{62}$. Collectively, these data indicate that MYCN hyperactivity through various means can contribute to WT through a multitude of mechanisms.

\section{Epigenetic modifiers}

A striking genotype-phenotype correlation is observed in epithelial-predominant WT, which is often driven by inactivating TRIM28 mutations (REFS ${ }^{63-66}$ ). Gene expression analyses have identified these tumours as more mature, post-induction tumours with excellent prognosis. TRIM28 is part of a chromatin silencing complex that has an important function in the repression of endogenous retroviral transcript in embryonic cells. Indeed, these tumours show strong induction of transcripts from repetitive elements, but the mechanistic links to oncogenic transformation in these tumours with otherwise few mutations remains to be established.

Besides TRIM28, studies have shown that a whole array of epigenetic regulators are potential drivers in WT. These regulators include REST, RERE, CHD4, KDM $3 B$, $B C O R$ and $B C O R L 1$, which are all components of large protein complexes with diverse enzymatic activities ${ }^{42,44}$. There is a spectrum of dominant and recessive as well as

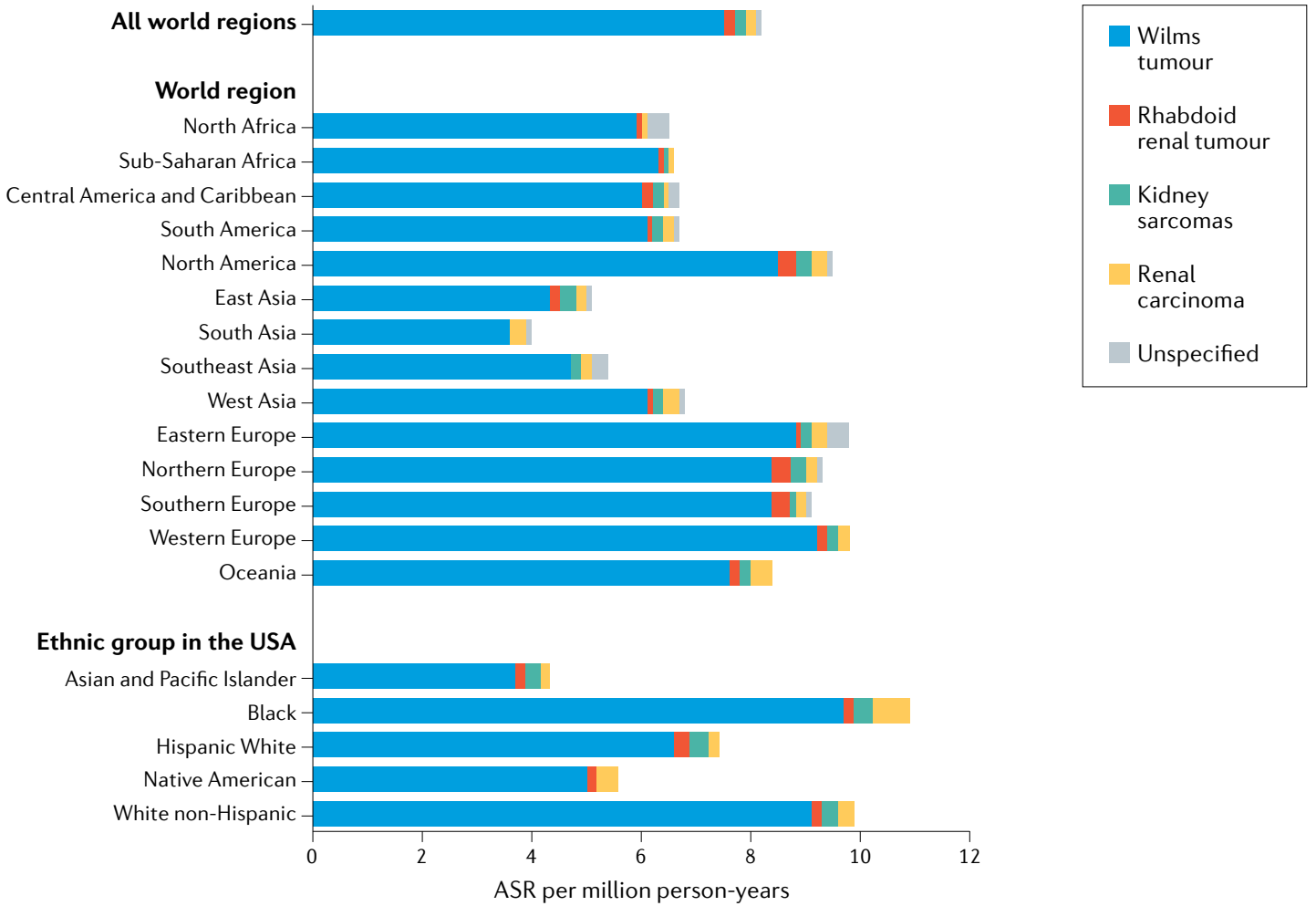

Fig. 3 | The incidence of Wilms tumour according to geographical area and ethnicity. Age-standardized incidence rates (ASRs) of renal tumours in children 0-14 years of age by world region and ethnicity, 2001-2010 ( $N=15,320)$. Unspecified, unspecified malignant renal tumours. Adapted from REF. ${ }^{2}$, CC BY 4.0. 
Table 1 | Heritable syndromes associated with an increased risk of Wilms tumour

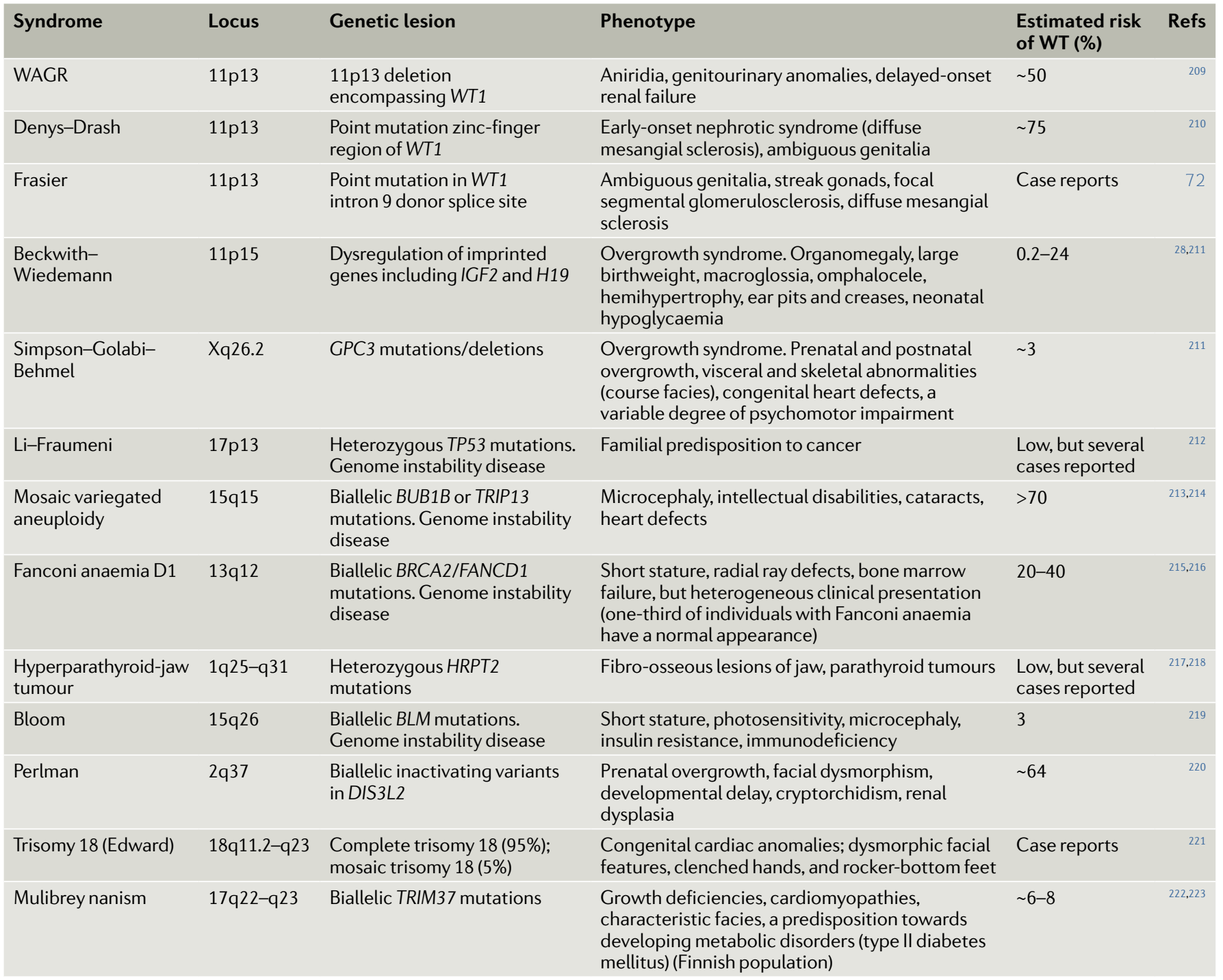

WT, Wilms tumour.

Chromothripsis

A catastrophic chromosomal

shattering event associated

with random rejoining.

Li-Fraumeni syndrome

An inherited autosomal

dominant cancer predisposition disorder that is usually

associated with abnormalities

in TP53 located on

chromosome 17p13.

Anaplasia

Cells with hyperchromatic, pleomorphic nuclei that are

three times larger than adjacent cells and have abnormal mitotic figures. Anaplasia is associated with a poor response to chemotherapy. truncating or missense mutations, some being heritable. Intriguingly, $B C O R$ is also the main culprit for driving clear cell sarcoma of the kidney, another childhood renal tumour. In this case the gene is not inactivated as in WT, but harbours small carboxy-terminal tandem duplications corresponding to $\sim 30$ amino acids that encompass the binding domains for PCGF1 and KDM $2 B$ as part of the polycomb repressive complex (PRC1) that controls, for example, mesodermal differentiation ${ }^{67,68}$.

\section{TP53 and anaplasia}

WTs generally have a low mutation load that increases with patient age, and karyotypes tend to be stable ${ }^{43,44}$. However, the mutation load is different in diffuse anaplastic WT, which frequently harbours oncogenic TP53 mutations. In most cases, the wild-type allele is lost and the cells are characterized by chromosomal instability including chromothripsis and deregulation of cell cycle and DNA repair genes ${ }^{59,69-71}$. TP53-mutated tumours exhibit strong positive p53 staining owing to the accumulation of the mutant protein, although a smaller fraction demonstrate negative staining due to null mutations. TP53 alterations are secondary events in WT progression, in line with WT being reported as a rare feature in Li-Fraumeni syndrome ${ }^{72}$.

Whether TP53 mutation confers an additional risk independent of the high-risk status of morphological anaplasia is still unknown. Several other genes that fall into the category of genome maintenance and repair, such as BRCA2, PALB2 and TRIP13, have been found to be mutated in $\mathrm{WT}^{73}$. Whether such mutations likewise increase mutation load or chromosomal aberrations remains to be determined, and no reports are available on aneuploidy yet.

\section{Nephrogenic rests}

The underlying genetic defects also have an impact on the presence of nephrogenic rests in the kidney that occur in $30-40 \%$ of patients $\mathrm{s}^{74,75}$. These precursor lesions are foci of embryonic renal cells that abnormally persist beyond 36 weeks of gestation. Nephrogenic rests are histologically and anatomically classified as either perilobar 
or intralobar ${ }^{74}$. WT1-related WTs frequently carry few intralobar nephrogenic rests, centrally located within or adjacent to the renal medulla, suggestive of an early developmental lesion. TRIM28-associated or BeckwithWiedemann syndrome-associated WTs tend to harbour perilobar nephrogenic rests in the adjacent kidney tissue rather than intralobar nephrogenic rests. These perilobar nephrogenic rests may even encompass the entire renal cortex in extreme cases. Although few samples have been assayed thus far, nephrogenic rests seem to carry even fewer mutations than their adjacent $\mathrm{WT}^{5,76,77}$.

\section{Bilateral Wilms tumour}

Almost one in ten children present with bilateral WT or bilateral disease (WT with nephrogenic rests or nephroblastomatosis visible on imaging in the contralateral kidney), especially in syndromic cases ${ }^{32}$. WT1 is the most prominent driver in these cases ${ }^{32,52}$, together with specific imprinting abnormalities at 11 p15 affecting IGF2, although neither explain the majority of cases. TRIM28 inactivation is also frequent in bilateral and familial tumours $^{64-66,73}$. Importantly, bilateral tumours can be due to early postzygotic founder mutations in somatic cells that emerge before the divergence of left and right kidney primordia ${ }^{5}$. Individual clones may expand to yield mosaic kidneys with molecular evidence of clonal (mosaic) nephrogenesis. Thus, it may be justified to compare bilateral or multifocal tumours with blood and surrounding normal kidney tissue as controls to differentiate putative germline mutations, postzygotic mosaic events or single tumours with metastatic disease.

\section{Heterogeneity and subclassification}

Molecular analysis has unveiled intratumoural heterogeneity of WTs, with either chromosomal copy number alterations or mutations, for example, in WTX or TP53, being present in only a fraction of cells as evidence of tumour evolution ${ }^{78}$. These differences may become clearer with single-cell or single-nucleus analyses, which already highlighted a great cellular diversity ${ }^{4}$. Even the main driver genes stratify WTs according to the age of the child (for example, TRIM28, WT1 in WT occurring at younger ages (generally $<2$ years of age), TP53 (generally $>4$ years of age) and Beckwith-Wiedemann syndrome occurring at older ages (3-4 years of age)), location of nephrogenic rests (intralobar versus perilobar) or histology (WT1 in stromal WTs, TRIM28 in epithelial WTs and TP53 in anaplastic WTs) (FIG. 5). Nevertheless, the majority of triphasic or blastemal-predominant WTs do not carry defining genetic alterations.

\section{Liquid biopsies}

Although WT represents $>80 \%$ of paediatric renal tumours, other intrarenal tumours exist that are important to differentiate as therapeutic approaches are markedly diverse ${ }^{31}$. These non-Wilms renal tumours are often characterized by rather specific molecular alterations (FIG. 5). These tumours may become amenable to liquid biopsy diagnostics

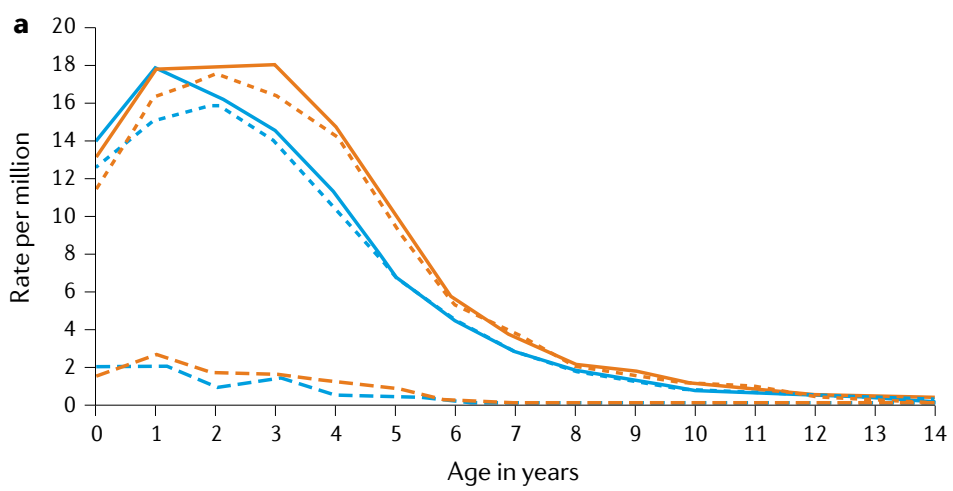

$$
\begin{aligned}
& - \text { Males }(n=6,606) \\
& - \text { Females }(n=7,232) \\
& --- \text { Unilateral males }(n=2,732) \\
& -=- \text { Unilateral females }(n=3,032) \\
& --- \text { Bilateral males }(n=261) \\
& --- \text { Bilateral females }(n=307)
\end{aligned}
$$
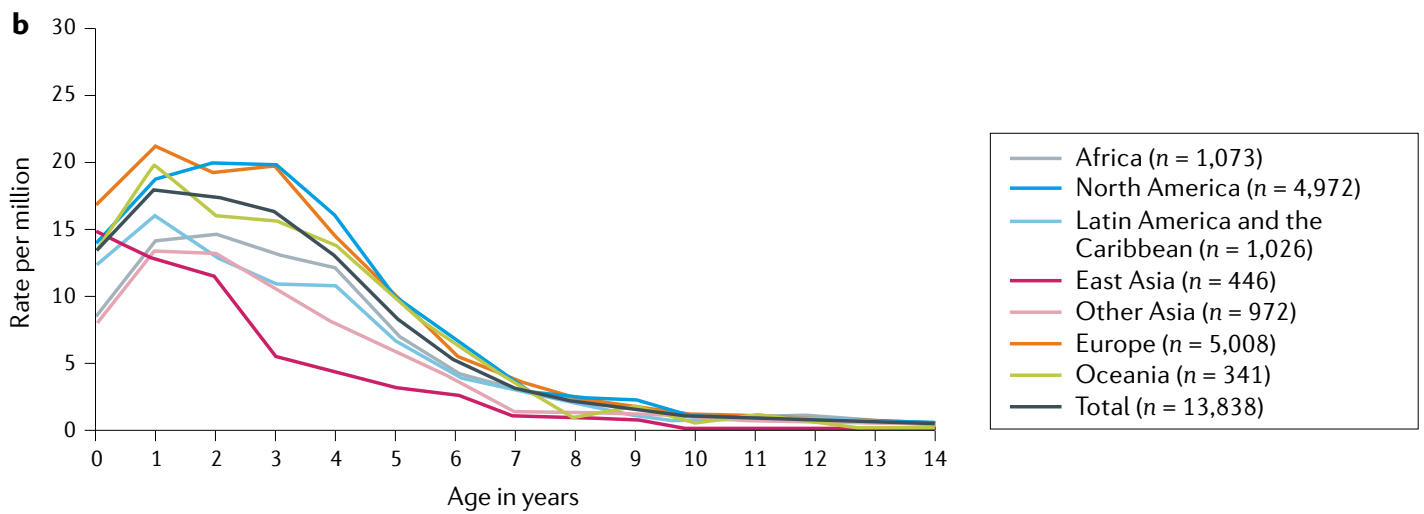

Fig. 4 | Age-specific incidence of Wilms tumour according to gender, laterality and geographical area. a | Age-specific incidence of Wilms tumour (WT) in children 0-14 years of age, all world regions combined, by sex $(N=13,838)$ and laterality $(N=6,396), 2001-2010$. ${ }^{a}$ Only the registries providing information on the laterality for at least 95\% of patients with WT are included. b | Age-specific incidence of WT in children 0-14 years of age by world region, 2001-2010 ( $N=13,838)$. Adapted from REF.2, CC BY 4.0. 
looking for diagnostic changes or entity-specific patterns of methylation ${ }^{79}$. In particular, if a neoadjuvant chemotherapy approach is planned and the clinical pattern is unusual, such tests will become helpful to rule out non-WT from the start, or to follow response to treatment during follow-up. The fact that patients with paediatric kidney tumours often have large amounts of circulating tumour DNA makes this approach rather promising ${ }^{80}$.

\section{Tumour models}

Modelling WT in the mouse has been difficult with only a few successful scenarios, the first using $W t 1$ ablation together with Igf2 upregulation ${ }^{81}$. Other researchers have

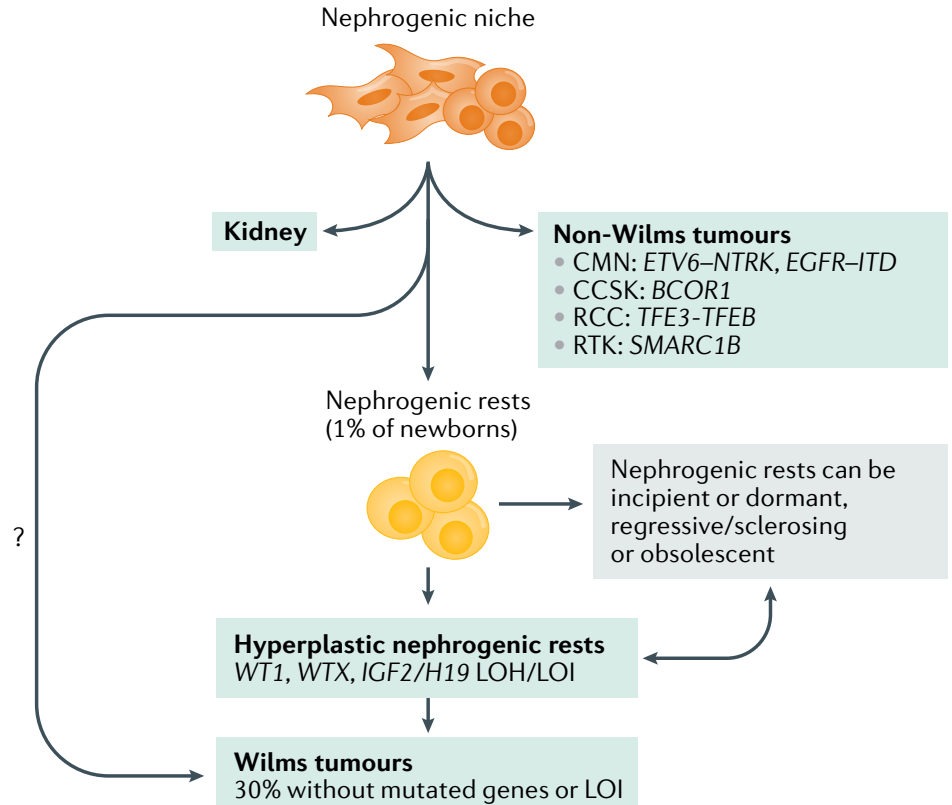

$30 \%$ without mutated genes or LOI

\begin{tabular}{|c|c|c|c|}
\hline $\begin{array}{l}\text { Stromal } \\
\text { WT1 (12\%) } \\
\text { CTNNB1 (15\%) }\end{array}$ & $\begin{array}{l}\text { Epithelial } \\
\text { TRIM28 (5\%) }\end{array}$ & $\begin{array}{l}\text { Regressive, mixed, blastemal } \\
\text { miRNA biogenesis (DROSHA, DGCR8, } \\
\text { DICER1, DIS3L2, XPO5, TARBP2) 15\%, } \\
\text { SIX1, SIX2 (5-10\%), MYCN (<10\%), } \\
\text { ARID1A, ASXL1, BCOR, BCORL1, } \\
\text { COL6A3, CTNNB1, FBXW7, MAP3K4, } \\
\text { MAX, MLLT1, NONO (<5\% cases) }\end{array}$ & $\begin{array}{l}\text { Anaplastic } \\
\text { TP53 (5\%, } \\
\text { secondary } \\
\text { event) }\end{array}$ \\
\hline
\end{tabular}

Fig. 5 | Biology of paediatric renal tumours. Cells deriving from intermediate mesoderm form the nephrogenic niche and develop into the various cell types of the normal kidney. Molecular alterations in these cells may result in diverse renal tumours: $\sim 90 \%$ being Wilms tumours (WTs) and $\sim 10 \%$ other primary renal tumours. In a paradigm of disrupted organ development eventually leading to tumorigenesis, remains of the multipotent nephrogenic zone of the fetal kidney may persist after birth and appear in up to $1 \%$ of routine infant autopsies as nephrogenic rests. The natural history and fate of nephrogenic rests is, however, uncertain. These cells may terminate their differentiation, or eventually regress and become sclerotic and obsolescent, whereas others can progress to form hyperplastic nephrogenic rests, with typical genetic changes. Nephrogenic rests are found in $>90 \%$ of patients with bilateral WTs and in $~ 30-40 \%$ of patients with unilateral sporadic WT. WTs are then characterized by the acquisition of additional genetic and epigenetic changes, some of them being quite specific for histological subtypes. The percentages indicate the frequency of mutation in sporadic cases. It is unclear whether WTs originate directly from nephrogenic blastema without progression through nephrogenic rest stages. CCSK, clear cell sarcoma of the kidney; CMN, congenital mesoblastic nephroma; LOH, loss of heterozygosity; LOI, loss of imprinting; miRNA, microRNA; RCC, renal cell carcinoma; RTK, rhabdoid tumour

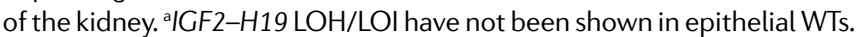

successfully employed Lin 28 overexpression or Dis $3 / 2$ mutation $^{82,83}$. On the other hand, prototypic Drosha mutations or Wtx deletions did not yield evidence of WT formation but led to either kidney agenesis or aberrant kidney development and functional impairment ${ }^{84,85}$.

Modelling efforts, including patient-derived xenografts $(\mathrm{PDX})^{86}$, can now be complemented with spheroid and organoid techniques to grow tumour cells in vitro for genetic and histological characterization and for therapeutic compound testing ${ }^{87-89}$. These models will become an invaluable resource to test novel agents in patients with relapse who respond poorly to conventional regimens, provided a time frame suitable for clinical feedback can be accomplished ${ }^{90}$.

\section{Diagnosis, screening and prevention Clinical presentation}

Most children with WT are asymptomatic at presentation and predominantly have a distended abdomen with a palpable mass ${ }^{91}$. Frequently, the parents notice such a mass during dressing or cuddling. Alternatively, WT is identified by the general practitioner or the paediatrician during a regular clinical assessment of a well child or a child with non-specific symptoms. WT usually reveals a non-tender, large flank mass, which does not move with respiration in contrast to splenomegaly. Approximately only one in five children have distinct symptoms; pain, haematuria, fever, hypertension, urinary tract infections, constipation and weight loss are among the most common complaints at presentation ${ }^{31,91}$. Although rare, symptoms related to metastases, such as dyspnoea (lung), abdominal pain (liver) or tumour thrombus in the renal vein or vena cava or varicocele, may occur ${ }^{92}$. Ultimately, a few children with severe subcapsular haemorrhage may present with rapid abdominal enlargement, anaemia and severe pain. Age at presentation is typically in the range 2-5 years and the incidence of WT in children $>10$ years is rare. In children with known predisposing syndromes, WT may be captured during routine screening and often at an earlier age or stage, and these children are even more likely to be asymptomatic than children without predisposing syndromes ${ }^{93}$.

In low-income countries (LICs), interactions between multiple factors usually contribute to a delayed diagnosis compared with diagnosis in high-income regions $^{94,95}$ (BOX 2). These factors include family or relatives' awareness of a possible cancer, contacting and arrival in primary care, health-care staff recognition of cancer and transfer to tertiary care. Furthermore, a much higher number of children in LICs have a distended abdomen due to other conditions, for example, malnutrition, parasitic infections and benign blood diseases than in high-income regions. Hence, identifying, differentiating and prioritizing investigations of the relatively few cases of WT is challenging. Moreover, the latency to diagnosis (patient interval and diagnostic interval) prolongs further, as diagnosis is not only dependent on the recognition by the family, but also by the lack of awareness by the primary care medical personnel and poor referral networks ${ }^{96}$. These factors result in a larger proportion of children presenting with symptoms, a large tumour volume, advanced local stage and 
Table 2 | The landscape of cancer genes that are potentially operative in Wilms tumorigenesis

\begin{tabular}{|c|c|c|c|c|c|}
\hline Gene & $\begin{array}{l}\text { Role in } \\
\text { tumorigenesis }\end{array}$ & Notes & $\begin{array}{l}\text { Reported } \\
\text { frequency }^{42}\end{array}$ & $\begin{array}{l}\text { Potential targeted therapeutic } \\
\text { approaches }\end{array}$ & Refs \\
\hline TP53 & $\begin{array}{l}\text { Tumour suppressor } \\
\text { gene }\end{array}$ & $\begin{array}{l}\text { Strongly associated with anaplasia; potential } \\
\text { driver of disease progression }\end{array}$ & $\begin{array}{l}\sim 5 \%(50-90 \% \\
\text { in DA) }\end{array}$ & $\begin{array}{l}\text { p53-specific biological targeting } \\
\text { agents }\end{array}$ & $69-71,90$ \\
\hline WTX & $\begin{array}{l}\text { Tumour suppressor } \\
\text { gene }\end{array}$ & Negatively regulates the Wnt pathway & 10-20\% & NA & 38,54 \\
\hline SIX1, SIX2 & $\begin{array}{l}\text { Implicated in renal } \\
\text { development }\end{array}$ & $\begin{array}{l}\text { Specificity for blastemal regions; association } \\
\text { with PLNR }\end{array}$ & $5-10 \%$ & NA & 43,44 \\
\hline MYCN & Oncogene & Copy number gain or specific P44L mutation & $\sim 15 \%$ & Directly targeting MYCN & $59,90,225$ \\
\hline $\begin{array}{l}\text { H19-IGF2 } \\
\text { locus }\end{array}$ & $\begin{array}{l}\text { Epigenetic } \\
\text { abnormalities at } \\
\text { the imprinted loci } \\
\text { on } 11 \mathrm{p} 15\end{array}$ & $\begin{array}{l}\text { LOI or loss of maternal allele (pUPD) at the BWS } \\
\text { imprinting centre } 1 \text { (IC1) leading to increased } \\
\text { IGF2 expression; association with PLNR }\end{array}$ & $\sim 50-80 \%$ & $\begin{array}{l}\text { Targeting of IGF2; methylation and } \\
\text { epigenetic targeting agents }\end{array}$ & $\begin{array}{r}38,43,44, \\
52,226\end{array}$ \\
\hline
\end{tabular}

Arm, armadillo; BWS, Beckwith-Widermann syndrome; COG, Children's Oncology Group; DA, diffuse anaplasia; dsRBD, double-stranded RNA-binding domain; ILNR, intralobar nephrogenic rest; LOI, loss of imprinting; miRNA, microRNA; NA, not available; PLNR, perilobar nephrogenic rest; pUPD, paternal uniparental disomy.

metastases in low-income regions than in high-income regions $^{14,24}$.

\section{Diagnosis, classification and staging}

Diagnosis of WT can be made reliably on histology, especially WTs in which all three characteristic components - blastemal, epithelial and stromal — are evident. These components may be mixed in any proportion, but WTs showing one or two components are not rare. Epithelial and stromal components may show different lines of differentiation and degrees of differentiation, resulting in a countless number of histological appearances (FIG. 6). WTs composed of only one component may represent a diagnostic challenge and ancillary techniques may be needed to establish the diagnosis ${ }^{97}$. However, no immunohistochemical markers or molecular biology findings are $100 \%$ specific for WT. In addition, preoperative chemotherapy, when used, alters the histological appearance of WT, and may result in marked tumour necrosis, or maturation of tumour components. Approximately $7-8 \%$ of WTs demonstrate anaplasia, defined as the presence of cells with hyperchromatic, pleomorphic nuclei that are three times larger than adjacent cells and have atypical mitotic figures ${ }^{98}$, and it may occur in any tumour component (blastemal, epithelial or stromal). The definition of anaplasia was further refined to specify whether the anaplasia is diffuse or focal based on the anatomical distribution of anaplastic cells within the tumour 99 . The diagnosis of focal anaplasia is based on the presence of clearly defined one or two foci showing the above-mentioned nuclear criteria with sharp demarcation within the primary intrarenal tumour and without evidence of anaplasia or prominent nuclear atypia (defined as nuclear unrest) in other areas. According to SIOP, up to two foci up to $15 \mathrm{~mm}$ in size is allowed for the diagnosis of focal anaplasia ${ }^{9}$ whereas according to COG, up to four foci up to $20 \mathrm{~mm}$ in size is allowed ${ }^{99}$. Diffuse anaplasia is defined as non-localized anaplasia, which may present in any of these situations: focal anaplasia with marked nuclear unrest in the non-anaplastic tumour; anaplasia beyond the tumour capsule; anaplastic cells in intrarenal or extrarenal vessels, renal sinus or extracapsular sites, in metastases, or in biopsy. Despite well-established criteria, anaplasia represents a diagnostic problem, with $\sim 30-50 \%$ discrepancy between institutional pathologists and central pathology review ${ }^{100,101}$. Anaplasia is very rare in the first 2 years of life, and increases after 4 years of age. Anaplasia is usually neither obliterated nor induced by preoperative chemotherapy.

As SIOP and COG have different treatment initial strategies, relevant differences exist in histological classifications of WTs between the two groups. The COG classification includes anaplastic (focal and diffuse) and non-anaplastic (favourable histology) WTs based on assessment of a chemonaive tumour after up-front surgery. The SIOP classification is based on the assessment of the percentage of preoperative chemotherapy-induced changes and viable tumour components, and includes three major WT risk groups: low-risk tumours (completely necrotic WT), high-risk tumours (blastemal type and diffuse anaplasia), and intermediate-risk tumours (all other types) (TABLE 3). To correctly subclassify the WT, the percentages of chemotherapy-induced changes and viable tumour components are assessed and taken into account ${ }^{9}$. COG has reported histology and outcomes in patients not eligible for up-front surgery using 


\section{Box 1 | Wilms tumour predisposition and driver genes}

Most genes implicated in Wilms tumorigenesis act in gene expression control and growth factor signalling. Approximately $50 \%$ of the genes can be present in mutant form in germline or constitutional DNA conferring increased Wilms tumour (WT) risk ${ }^{51}$.

Kidney development

CTNNB1, SIX1, SIX2, WT1 a

Transcriptional machinery

CDC73a, CREBBP, CTR9 ${ }^{a}$, FBXW7 ${ }^{a}, M A X, M L L T 1$, MYCN

Chromatin biology or epigenetic modifiers

ARID1A, ASXL1 ${ }^{\mathrm{a}}, \mathrm{BCOR}(\mathrm{L1})^{\mathrm{a}}, \mathrm{BRD} 7, \mathrm{CHD} 4, \mathrm{HDAC} 4, \mathrm{KDM}_{3} \mathrm{~B}^{\mathrm{a}}, \mathrm{RERE}, \mathrm{REST} \mathrm{T}^{\mathrm{a}}, \mathrm{TRIM}_{2} 8^{\mathrm{a}}$, TRIM37

MicroRNA processing and RNA metabolism

DGCR8, DICER1 ${ }^{a}$, DIS3L2ª , DROSHA, LIN28B, NONO, NYNRINª, TARBP, XPO5

Growth factor signalling

ACTB, AMER1, FGFR1, GPC3 ${ }^{\text {a }, ~ I G F 2 ~(B W S-I C 1) ~ a ~, ~ M A P 3 K 4, ~ N F 1 ~}{ }^{\text {a }}$, PIK3CA

Genome maintenance

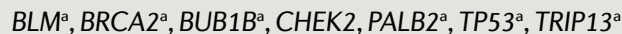

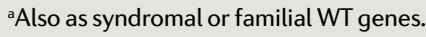

the SIOP post-chemotherapy histological classification system but to date has not used this system to guide subsequent treatment in patients with a unilateral tumour ${ }^{102}$. The staging criteria between COG and SIOP also differ, making a direct comparison of outcomes stage-by-stage difficult (Supplementary Table 1).

Diagnostic imaging. Abdominal ultrasonography is efficient and globally the most available means of investigating suspected $\mathrm{WT}^{103}$. Ultrasonography provides information about the organ of origin, extension into the renal and inferior cava veins or urinary collecting system, the contralateral kidney and associated urogenital abnormalities, and may identify liver or lymph node metastases. In resource-limited regions, ultrasonography is sufficient for abdominal staging and can be complemented by chest plain radiography, recognizing that plain radiography may miss smaller pulmonary lesions (typically $<1 \mathrm{~cm})^{95,104}$. In better-resourced settings, crosssectional imaging is usually undertaken preoperatively with abdominal CT or $\mathrm{MRI}^{105}$. The main drawback of CT is radiation exposure, but the procedure is rapid, allows continuous imaging of the chest and abdomen, has moderate specificity for detection of preoperative spill, may help distinguish nephrogenic rests from WT and gives excellent pulmonary detail ${ }^{106-108}$. It is noteworthy that COG and SIOP incorporate centrally reviewed CT identification and response to therapy of lung nodules into current risk stratification treatment algorithms $\mathrm{s}^{10,109}$. The main hurdle of abdominal MRI is that moderate to deep sedation is often required in young children but it provides excellent organ details in those with bilateral disease or liver metastases. Abdominal MRI is preferentially recommended for better assessment of potential nephrogenic rests and their distinction from true WT, and by SIOP to attempt to correlate apparent diffusion coefficient mapping with histopathology prediction after preoperative chemotherapy ${ }^{105,110}$.

Fluorodeoxyglucose (FDG) PET is not routinely used for imaging $\mathrm{WT}^{105}$. Bone scan or cross-sectional imaging of other sites is reserved for patients with signs or symptoms suspicious for distant extrapulmonary metastases. Non-pulmonary and non-hepatic metastatic disease is very rare at primary diagnosis of non-anaplastic WT and is more likely observed in anaplastic WT, clear cell sarcoma of the kidney, malignant rhabdoid tumour and renal cell carcinoma, or at WT relapse ${ }^{111-113}$.

Laboratory testing. Baseline blood work should be performed to confirm adequate renal function, support subsequent chemotherapy and rule out acquired von Willebrand's disorder, which although uncommon may be associated with substantial bleeding risks and can be pre-emptively managed ${ }^{114}$.

SIOP diagnostic algorithms recommend percutaneous image-guided coaxial core needle biopsy through a retroperitoneal approach in patients 7 years of age or older or in patients with imaging findings unusual for WT (psoas muscle infiltration, numerous calcifications, vessel encasement or massive lymphadenopathy $)^{10,115,116}$. The currently used cut-off of 7 years to consider a biopsy is under revision, and based on epidemiological data showing the peak incidence of WT versus other non-WTs ${ }^{2}$, a new consensus towards raising the age threshold for biopsy providing there are no other atypical presenting features is forming ${ }^{105,115}$. COG recommends that primary nephrectomy should be strongly considered in all patients, but if not feasible, open or Tru-Cut needle biopsy should be undertaken with a minimum of 10-12 cores. Notably, needle biopsy cannot reliably distinguish WT from nephrogenic rests, and often misses anaplasia ${ }^{115}$.

Patients with syndromic features should be referred to medical genetics for counselling and possible testing. Circulating blood or urine tumour DNA is being explored for diagnostic and response or relapse assessment but is not yet standard of care ${ }^{80,117,118}$.

\section{Prognosis and prognostic features}

It is important to recognize that prognostic markers must be interpreted in the context of the accompanying treatment regimen. This principle is relevant to WT as COG advocates for immediate nephrectomy in most patients, whereas SIOP advocates for preoperative chemotherapy ${ }^{119}$. Thereafter, prognostic factors used for clinical treatment stratification differ between COG and $\mathrm{SIOP}^{120,121}$. According to both groups, tumour histology and stage are key prognostic indicators, although applied differently and together with other factors in clinical practice. Diffuse anaplasia is regarded as a high-risk tumour by COG and SIOP, whereas focal anaplasia is regarded as an intermediate-risk tumour by SIOP but as a high-risk tumour by COG. A blastemal-type WT after preoperative chemotherapy is also regarded by SIOP as a high-risk tumour and a completely necrotic-type WT as a low-risk tumour ${ }^{122}$ (TABLE 3). Similarly, staging criteria are also different; for example, according to COG, any tumour biopsy results in upstaging to local stage III, whereas according to SIOP, fine-needle aspiration and percutaneous core needle biopsy are ignored for staging purposes ${ }^{31}$, and according to SIOP, the presence of necrotic tumour or chemotherapy-induced changes in 
the renal sinus, renal veins and/or the perirenal fat is not a reason for upstaging to stage II $^{9}$ (Supplementary Table 1). Some SIOP criteria have undergone important changes in comparison with the previous SIOP-2001 trial and study criteria. For example, in the current SIOP protocol, the presence of non-viable tumour or chemotherapy-induced changes only at a resection margin is not regarded as stage III ${ }^{9}$.

Other prognostic factors according to SIOP include tumour histological response to preoperative chemotherapy and tumour volume $(>500 \mathrm{ml})$ after chemotherapy for certain WT types. Additional prognostic factors according to COG include age, tumour weight and biomarkers or tumour biology, that is, loss of heterozygosity at chromosomes $1 \mathrm{p} / 16 \mathrm{q}$, loss of heterozygosity at chromosome $11 \mathrm{p} 15$, and gain at chromosome $1 \mathrm{q}^{121}$. For both groups, response of lung metastases to neoadjuvant chemotherapy indicates chemosensitivity and dictates

Box 2 | Challenges and priorities for managing patients with Wilms tumour in low-income and middle-income countries

\section{Challenges}
- Highly constrained health-care budgets resulting in insufficient paediatric oncologists, surgeons, anaesthetists and pathologists; shortage of chemotherapeutic agents (which leads to incomplete Wilms tumour (WT) treatment); limited or lacking infrastructure and facilities for imaging and radiation therapy.
- Lack of high-quality specialized paediatric surgical training to perform complex operations (WT with intracaval extension, nephron-sparing surgery).
- Inadequate reporting or data collection within national or hospital registries precludes accurate outcomes assessment.
- Inadequate specialist cancer services.
- Late clinical presentation (delay in diagnosis) owing to family's or relatives' reduced awareness about cancer; contacting and arrival to primary care; health-care staff recognition of cancer (a much higher number of children in low-income countries have a distended abdomen than in high-income countries owing to many other non-malignant conditions; thus it is challenging to differentiate and prioritize investigations for the relatively few children with WT).
- Many patients are diagnosed with already advanced or metastatic tumours.
- Toxicity from surgery and/or chemotherapy can increase mortality and contribute to treatment abandonment.
- Malnutrition is a major concern for higher drug toxicity and treatment-related death.
- Burden of associated comorbidities (infections).
- Patient quality of life largely unrecognized and unprioritized.

\section{Priorities and areas for improvement}

- Comprehensive registries are the first step to appropriate resource allocation according to local needs and to the monitoring of improvement.

- Earlier diagnosis through increased education among primary health providers concerning WT diagnosis, and parent education on healthy living and concerning symptoms.

- Adapted treatment regimens to accommodate frail children, to reduce toxicity, and to face specific (temporary or permanent) drug regimen shortages.

- Nutritional programmes, best with locally available calorie-dense foods and fortifiers.

- Implementation of family education programmes may increase compliance with cancer care reducing abandonment.

- Twinning programmes (pairing of hospitals in resource-limited countries with hospitals in developed countries) to improve local medical expertise and education.

- Clinical trials answering locally relevant questions (such as prognostic factors).

- Prioritizing resources to focus on curable clinical situations.

- Palliative care as the main priority for advanced malignancies. the intensity of subsequent treatment; for example, if lung lesions are not present at 6 weeks after induction chemotherapy, radiotherapy can be omitted in some patients ${ }^{109,123}$.

Although the SIOP and COG strategies differ in their upfront treatment approach, they lead to similar overall survival rates of $\sim 90 \%^{7,39,124}$. Patients with stage IV anaplastic WT and/or blastemal type WT have substantially poorer outcomes, with an overall survival rate of $<50 \%$ despite very intensive therapy ${ }^{125,126}$.

Despite the good prognosis in most children with WT, $\sim 20 \%$ of patients will relapse, predominantly within 2 years of diagnosis ${ }^{113,127,128}$. The overall survival rate after relapse is $\sim 50 \%$ but varies considerably according to the initial treatment received (which in turn reflects initial tumour stage and histology), time to relapse, site of relapse, and patient age ${ }^{113,129,130}$. Surveillance with abdominal ultrasonography and chest plain radiography are offered, and patients with asymptomatic relapse detected by surveillance seem to have better outcomes ${ }^{113}$. Evidence from COG shows a lack of benefit in terms of longer survival after relapse if CT imaging had been used instead of plain radiography and ultrasonography in follow-up surveillance ${ }^{128}$. SIOP data also suggest that surveillance beyond 2 years after completion of therapy has a low yield because of the extremely low relapse rate thereafter ${ }^{113}$.

\section{Screening}

Genetic testing in children with cancer but also in other (potentially) unhealthy children presenting with certain abnormalities or syndromes is emerging. This testing includes formalized national and regional whole-exome or genome sequencing programmes to detect cancer predisposition in many high-income regions. Accordingly, both novel genes and syndromes associated with WT are revealed and additional children with an increased risk of developing WT are identified, expanding the criteria for screening programmes ${ }^{131}$. Regular screening for early diagnosis in children with a known WT predisposition syndrome has been reported to detect smaller and lower-stage tumours but robust evidence is lacking regarding the balanced clinical benefits ${ }^{93}$. In addition, the benefits should outweigh the costs and burden. The latter is reflected in the different thresholds for performing screening, which typically varies between $1 \%$ and $5 \%$ for the childhood risk of developing $\mathrm{WT}^{29}$.

Screening is typically offered to children with various cancer predisposition syndromes, such as WT1-related syndromes and Beckwith-Wiedemann syndrome or isolated hemihypertrophy (with at least one BeckwithWiedemann syndrome feature). Renal ultrasonography is the recommended screening modality, as it avoids radiation and does not require anaesthesia in young children. The screening interval is every 3 months based on the rather rapid growth rate of the tumour and imaging should be performed by an experienced paediatric ultrasonographer ${ }^{93}$. Screening should start when the WT predisposition is established and should continue, irrespective of the underlying condition, until the child is approximately 7 years old. At this age, the risk of WT development is greatly reduced ${ }^{29}$. 
The purpose of WT screening is to enable early NSS, to give less-intensive (that is, less-toxic) chemotherapy, and to avoid radiotherapy. Patients with predisposition syndromes may develop metachronous WT in the contralateral kidney. Hence, the aim is, on balance, to preserve maximal kidney function and ultimately avoid end-stage renal disease whilst still maintaining oncological control. Genetic testing, screening and NSS in LICs are rarely available and consequently, more children progress and succumb to end-stage renal diseases in low-income settings with limited options for dialysis and/or renal transplantation than in high-income regions ${ }^{132}$. High-income regions are researching the potential for (epi)mutation detection in circulating tumour DNA for early diagnosis; however, this detection technique is not yet ready to be used as an alternative to surveillance with ultrasonography in clinical practice $^{117,118,133}$.
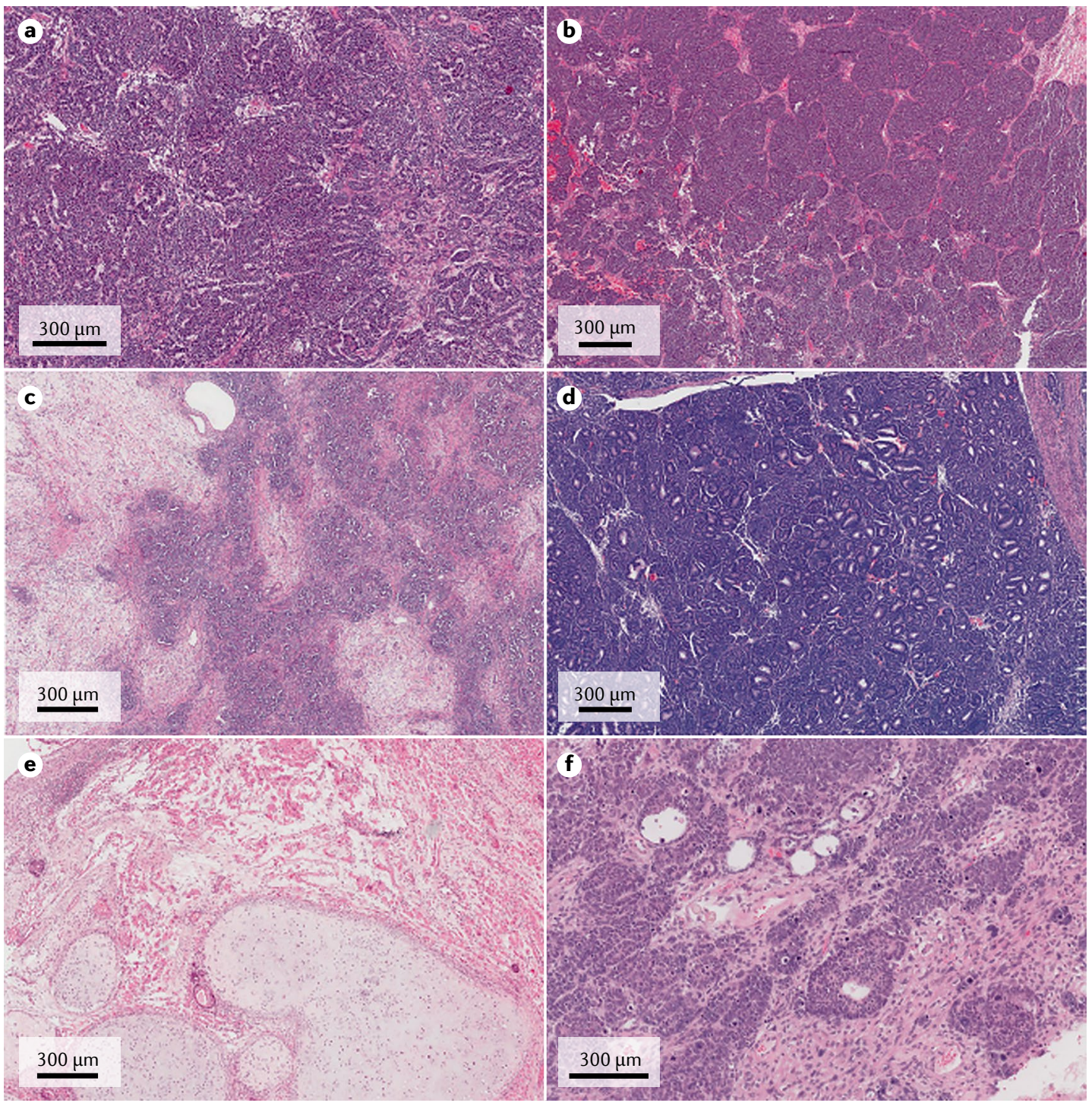

Fig. 6 | Different histological patterns of Wilms tumour. a Mixed type, with the blastemal and epithelial component. b | Blastemal type. $\mathbf{c}$ | Mixed type consisting of the mature epithelial and stromal components. $\mathbf{d}$ |Epithelial type composed of moderately differentiated tubules. e $\mid$ Stromal type with heterologous elements including cartilage and skeletal muscle. f | Anaplasia in Wilms tumour with atypical mitoses, nuclear enlargement and hyperchromasia. 
Table 3 | Risk classification according to SIOP Renal Tumour Study Group

\begin{tabular}{|c|c|c|}
\hline Tumour type & For pretreated patients & $\begin{array}{l}\text { For patients undergoing } \\
\text { primary nephrectomy }\end{array}$ \\
\hline $\begin{array}{l}\text { Low-risk } \\
\text { tumours }\end{array}$ & $\begin{array}{l}\text { Congenital mesoblastic nephroma } \\
\text { Cystic partially differentiated WT } \\
\text { Completely necrotic WT }\end{array}$ & $\begin{array}{l}\text { Congenital mesoblastic } \\
\text { nephroma } \\
\text { Cystic partially differentiated WT }\end{array}$ \\
\hline $\begin{array}{l}\text { Intermediate- } \\
\text { risk tumours }\end{array}$ & $\begin{array}{l}\text { WT epithelial type } \\
\text { WT stromal type } \\
\text { WT mixed type } \\
\text { WT regressive type } \\
\text { WT focal anaplasia }\end{array}$ & $\begin{array}{l}\text { Non-anaplastic WT and its } \\
\text { variants } \\
\text { WT focal anaplasia }\end{array}$ \\
\hline $\begin{array}{l}\text { High-risk } \\
\text { tumours }\end{array}$ & $\begin{array}{l}\text { WT blastemal type } \\
\text { WT diffuse anaplasia } \\
\text { Clear cell sarcoma of the kidney } \\
\text { Rhabdoid tumour of the kidney } \\
\text { Renal cell carcinoma }\end{array}$ & $\begin{array}{l}\text { WT diffuse anaplasia } \\
\text { Clear cell sarcoma of the kidney } \\
\text { Rhabdoid tumour of the kidney } \\
\text { Renal cell carcinoma }\end{array}$ \\
\hline
\end{tabular}

viable component of the resected tumour. On the other hand, the COG approach of upfront nephrectomy allows immediate histological diagnosis, molecular analysis of tumour samples unaltered by chemotherapy, and drug-naive local staging assessment (such as the presence of tumour spill or lymph node involvement). This knowledge can identify a subset of children with very low-risk tumours who may be treated with nephrectomy alone ${ }^{136}$. Each approach has its pros and cons, yet survival rates are similar with an overall survival rate of $>90 \%$. According to both groups, the management of WT incorporates risk-based adjuvant chemotherapy and radiotherapy informed by multiple prognostic factors ${ }^{6}$ (Supplementary Table 2).

\section{COG perspective}

COG has a recommended strategy of primary nephrectomy for unilateral renal masses in patients without WT predisposition (achievable in $>90 \%$ of patients) or failing feasibility of nephrectomy, core needle or open biopsy to guide subsequent therapy ${ }^{135}$. Exceptions to upfront biopsy is patients with bilateral disease or patients with a bilaterally predisposed syndrome who should receive neoadjuvant chemotherapy (without biopsy) with the aim of preserving renal units, with surgery at $6-12$ weeks after initiation of chemotherapy ${ }^{137,138}$. The primary surgery using a transabdominal or thoracoabdominal approach allows accurate prechemotherapy staging including assessment of chemotherapy-naive histology and prognostic molecular testing. Essential surgical tasks in completing a tumour-related nephrectomy include avoidance of tumour spill, ipsilateral hilar and regional lymph node sampling, and assessment and control of extrarenal tumour extension including the renal vein and ureter ${ }^{139-142}$. Less conventional approaches such as laparoscopic nephrectomy, partial nephrectomy and split renal techniques may be carefully considered in patients with selected small tumours and in expert hands but at this point is confined to a small number of patients $^{143-146}$.
Chemotherapy is a mainstay of adjuvant therapy except in very low-risk tumours (defined as stage I, favourable histology WT, <550g with negative lymph nodes and no syndromic features) where observation alone following nephrectomy may be sufficient, especially in the absence of loss of heterozygosity at chromosome 11p15 (REF. $\left.{ }^{147}\right)$. Based on COG staging, the bulk of patients with favourable histology WT without certain adverse biomarkers receive regimen EE4A (vincristine and actinomycin D for 18 weeks) for stage I and II, or regimen DD4A (vincristine, doxorubicin and actinomycin D for 24 weeks) for stage III and stage IV favourable histology WTs ${ }^{102,109,148-150}$. COG recommends the use of CT imaging to identify lung metastases, although it is recognized that up to one-third of lesions $<1 \mathrm{~cm}$ in diameter may be benign nodules. Biopsy of lung nodules is encouraged if there is any doubt about the nature of the lesion. In addition, round, non-calcified lung nodules not in a fissure visible on chest CT are considered stage IV, regardless of size, unless histologically proven not to be $\mathrm{WT}^{102}$. The COG study AREN0533 demonstrated that $\sim 40 \%$ of patients have complete resolution of pulmonary metastases after 6 weeks of three-drug induction therapy (regimen DD4A) and of these patients, those with tumours without 1q gain can safely have radiation omitted $^{109}$. Patients with an incomplete response of lung nodules after 6 weeks of DD4A chemotherapy receive whole-lung irradiation and are escalated to chemotherapy regimen $\mathrm{M}$.

In the setting of loss of heterozygosity at $1 \mathrm{p} / 16 \mathrm{q}$, evidence shows that intensifying therapy to regimen DD4A for stage I and II or to regimen M (DD4A + cyclophosphamide or etoposide) for stage III and IV improves event-free survival outcome ${ }^{39,151}$. Patients with diffuse anaplastic tumours seem to benefit from a multiagent regimen UH-2 (REF. ${ }^{125}$ ). This regimen is associated with considerable toxicity and further modifications are currently being tested in COG protocol AREN1921 (NCT04322318) (Supplementary Table 3). A variety of strategies for salvage treatment in patients with relapse are used based on risk. Patients with low-risk relapse are usually managed with stratum B with an expected outcome of $\sim 71 \%$ event-free survival rate ${ }^{152}$ and patients with higher-risk relapse are typically managed with regimen $\mathrm{C}^{153}$ or ICE (ifosfamide, carboplatin and etoposide) with an expected outcome of $\sim 42 \%$ event-free survival rate. Some centres use autologous bone marrow transplantation as consolidation therapy in patients with a high-risk tumour but this strategy has never been the subject of a randomized trial to confirm efficacy ${ }^{154}$. A detailed summary of the impacts of the first generation of COG studies on WT was published in 2021 and forms the basis of standard management approaches in the COG for those patients not participating in a research study ${ }^{155}$.

Newer COG research protocols are testing further refined chemotherapy algorithms incorporating stage, lymph node status, additional somatic molecular biomarkers, cardioprotection with dexrazoxane and new agents $^{40,102,125,151}$. WT is highly radiosensitive; ${ }^{156}$ radiation therapy is utilized for the regional management of stage III or IV favourable histology WT, and relapsed and anaplastic WT. COG protocols are incorporating 
intensity-modulated radiation therapy ${ }^{157}$ with doses ranging from 10.6 to $30.6 \mathrm{~Gy}$ depending upon residual tumour and site ${ }^{156,158}$. National Cancer Cooperative Network ${ }^{159}$ guidelines provide further detailed management guidelines and recommend that all children with renal tumours participate in a clinical trial.

\section{SIOP perspective}

According to the SIOP strategy, all patients $>6$ months of age with suspected WT receive either 4 weeks of preoperative chemotherapy with actinomycin $\mathrm{D}$ and vincristine (if localized) or 6 weeks of actinomycin D, vincristine and doxorubicin (if metastatic). The SIOP-9 trial showed no advantage on downstaging to more stage I tumours nor on reducing intraoperative tumour rupture by further prolonging the prenephrectomy regimen to 8 weeks ${ }^{160}$. The SIOP approach accounts for the risk of misdiagnosis of WT by recommending upfront nephrectomy in infants $<6$ months of age, and percutaneous core needle biopsy in older children ( 7 years of age and older) or children with uncertain clinical pictures ${ }^{115}$.

Radical nephrectomy is regarded as standard in most patients with unilateral WT; however, the systematic use of preoperative chemotherapy may extend NSS opportunities in selected patients with unilateral non-syndromic tumours $^{134}$ to maximize preservation of renal function in patients. Following surgery, patients are stratified into three risk groups according to the histopathological features of the tumour ${ }^{122}$ (TABLE 3); the histological risk group together with tumour stage is used to direct the intensity of adjuvant chemotherapy and the need for radiotherapy (Supplementary Tables 2 and 4).

The regimen used in the experimental arm of the SIOP-2001 trial has been adopted as the new standard regimen for most patients with stage II-III intermediaterisk histology $\mathrm{WT}^{7,10}$. This regimen consists of 27 weeks of postoperative treatment with vincristine and actinomycin D without doxorubicin. This schedule resulted in a non-significant decrease in event-free survival and had no effect on overall survival when randomly compared with the historical standard arm of 27 weeks of these two drugs (vincristine and actinomycin D) plus five doses of doxorubicin at $50 \mathrm{mg} / \mathrm{m}^{2}$ (REF.7). The use of doxorubicin in patients with intermediate-risk stage II-III tumours is currently recommended only in those with non-stromal or non-epithelial large-volume tumours (that is, $\geq 500 \mathrm{ml}$ after prenephrectomy chemotherapy), based on a post hoc analysis of patients in the SIOP 2001 trial $^{10}$.

Radiotherapy to the flank is administered to patients with stage II WT with diffuse anaplasia or stage III WT (intermediate-risk and all high-risk), and doses range from 14.4 to $25.2 \mathrm{~Gy}( \pm 10.8 \mathrm{~Gy}$ boost only for macroscopic residual disease $)^{10,161}$. To decrease organ toxicity whilst preserving oncological outcome, the conventional approach of flank irradiation is currently being adapted into a guideline for highly conformal image-guided flank target volume delineation ${ }^{162}$.

For metastatic disease, CT-only nodules are treated as metastases in the current SIOP protocol if they have a transverse diameter of $\geq 3 \mathrm{~mm}$ and imaging appearance suspicious for metastatic nodules after centralized radiological review ${ }^{10}$. Following a standard 6-week three-drug preoperative regimen, $61-67 \%$ of patients have complete metastatic response before nephrectomy ${ }^{123}$. Afterward, current SIOP guideline advise stratifying patients to adjuvant regimens consisting of either vincristine plus increasing cumulative doses of doxorubicin (in the range $150-250 \mathrm{mg} / \mathrm{m}^{2}$ ) or a four-drug regimen including etoposide, carboplatin, cyclophosphamide and doxorubicin (cumulative dose $300 \mathrm{mg} / \mathrm{m}^{2}$ ). In patients with remaining lung nodules, metastasectomy and histological confirmation of metastasis is advised. Stratification is based on the local stage of the primary tumour, histology of the primary tumour and the metastatic tumour (if resected), the size of metastases, and their response to preoperative chemotherapy and surgery (Supplementary Tables 2 and 4).

Pulmonary radiotherapy is administered for lung metastases lacking complete response by postoperative week 10. Evidence suggests that the majority of patients achieving a complete response after induction chemotherapy with or without surgery have a satisfactory survival probability even without radiotherapy to the lungs (5-year event-free survival 84\%, 5-year overall survival $92 \%)^{123}$. Patients with viable metastases at surgery or high-risk histology of the primary tumour receive radiotherapy to the lungs.

Patients with metastatic and high-risk disease are a rare subgroup with a dismal prognosis, justifying testing of novel and more intensive regimens in first-line therapy ${ }^{126}$. Combinations of vincristine, irinotecan, cyclophosphamide, carboplatin, etoposide and doxorubicin, followed by high-dose melphalan and autologous stem cell rescue, are currently being explored by the SIOP-RTSG ${ }^{10,126,130}$.

For bilateral tumours, SIOP guidelines aim to limit preoperative chemotherapy to a maximum of 12 weeks, with time intervals for evaluation to 6 weeks, also comparable with the COG approach. In order to maximize the possibility of bilateral NSS, an approach using carboplatinetoposide in patients with an unsatisfactory response to vincristine-actinomycin D is under evaluation ${ }^{10}$. Adjuvant postoperative treatment guidelines generally follow the same principles as for unilateral WT.

\section{Low-income and middle-income regions}

Survival in low-income and middle-income countries (LMICs) is much lower than in high-income regions, with overall survival rates ranging from $11 \%$ in Sudan to $46 \%$ in Malawi ${ }^{163-166}$. In LMICs factors that reduce the likelihood of a good outcome include delay in diagnosis leading to advanced disease at diagnosis ${ }^{94,167}$, lack of diagnostic services, insufficient trained personnel, chemotherapy and radiation ${ }^{14,95,168,169}$, misdiagnosis ${ }^{167}$ and abandonment of therapy ${ }^{14,169}$ (BOX 2). Mortality is higher than in high-income regions owing to toxicity from surgery and/or chemotherapy, coupled with malnutrition ${ }^{167,170-172}$. Addressing these psychosocial issues and malnutrition (chronic and acute) may significantly improve outcomes with time $e^{95,170,173}$. As experience is gained in these countries, there is a need for support for the development of local priorities and for improvement in the availability of curative therapies and palliative care. 
Trained physicians, nurses and ancillary personnel are central in providing quality care. The WHO Global Initiative for Childhood Cancer developed the framework of care CureAll to provide early diagnosis networks for referral to centres of excellence, to introduce childhood cancer to the Universal Health Coverage schemas and Cancer Control Plans, to introduce cancer and supportive care regimens of care, and to evaluate and monitor schemas to measure progress. These strategic plans are coupled with enabling actions - advocacy, leveraged financing and linked governance ${ }^{174}$.

The SIOP approach with prenephrectomy chemotherapy provides the optimum and safest strategy in resource-limited settings. For large abdominal tumours $(>500 \mathrm{ml})$, upfront surgery is associated with a high risk of surgical complications, tumour rupture and infection $^{175}$. Patients with severe malnutrition may have decreased clearance of chemotherapy and dose adjustment may be necessary with parallel monitoring of liver function and recovery of myelosuppression ${ }^{172,176}$. In a preoperative chemotherapy scenario, close attention to the interpretation of pathology according to the SIOP risk classification is key to correct selection of postoperative treatment intensity, but requires specifically trained pathologists ${ }^{9,10}$.

In LMICs, almost no clinical trials are available, with limited data and information on outcomes ${ }^{177}$. Encouraging prospective registration studies and participation in clinical trials has the benefit of building expert clinical capacity, improving facilities and funding treatment and associated costs with the effect of improved survival ${ }^{177}$, all according to the local sustainability and capacity building ${ }^{178}$.

As an example, the Collaborative WT Africa Project is a multinational prospective clinical study open in seven sub-Saharan countries, which have registered prospective outcomes by implementing the SIOP adapted treatment regimen for $\mathrm{WT}^{164,179,180}$. A minimum requirement of ultrasonography of the abdomen was used for diagnosis. The guidelines recommended preoperative chemotherapy followed by surgery and further chemotherapy, coupled with nutritional rescue. The preoperative treatment included either a 4-week twodrug (vincristine and actinomycin D) or a 6-week three-drug (vincristine, actinomycin $\mathrm{D}$ and doxorubicin) regimen depending on the presence of local or metastatic disease, respectively ${ }^{104}$. Prolongation of preoperative chemotherapy was an option in patients with a large tumour volume. Patients weighing $<12 \mathrm{~kg}$ or with severe acute malnutrition were given two-thirds of the calculated dose of the chemotherapy ${ }^{164}$. The goal was then to achieve safe nephrectomy with lymph node sampling in patients with improved clinical and nutritional conditions, and tumour shrinking, which are all related to a reduced incidence of intraoperative morbidity. The postoperative chemotherapy aimed to follow the standard SIOP guidelines, but with spacing the administration of vincristine every 3 weeks at a dose of $2 \mathrm{mg} / \mathrm{m}^{2}$ as also used for children $>1$ year of age in specific phases of some COG regimens (capped at $2 \mathrm{mg}$ absolute dose), with the purpose of reducing the burden of frequent travel to hospital ${ }^{181}$. Acknowledging deficits in radiotherapy provision (lacking across most of the African network), radiation therapy was used only in Kumasi and Accra, Ghana, for metastatic disease and for stage III abdominal tumour ${ }^{179}$.

The lessons learnt from this structured guideline was the need for team members to work according to a shared vision, mission and principles ${ }^{178}$. The importance of using local site leaders to set the priorities for a successful clinical trial and keeping processes as simple as possible for data completeness was also appreciated. Good communication, transparency and trust was found to be the cornerstone for successful local implementation of a multinational clinical trial in LMICs.

Just as clinical investigation is the cornerstone for best therapies and practices in high-income regions, research is needed to address the best therapy in different settings (better if tuned to prognostic indicators that have been studied and validated in the local context), best practices, and quality data to further improve outcomes ${ }^{164,165}$. Multidisciplinary care meetings with mentors improve management and experience of the local and regional groups.

\section{Long-term complications}

Despite the greatly improved therapy for WT over time, survivors still report a high frequency (25\%) of severe chronic health conditions in adult life ${ }^{15,16,182,183}$. Patients with WT have a higher risk of death than the general population. In an analysis of children enrolled in the NWTS group between 1969 and 1995, the standardized mortality ratio was 24.3 during the first 5 years after diagnosis, but remained increased for $>20$ years after diagnosis (standardized mortality ratio 4.3 ) $^{184}$. Although the primary tumour remained the most frequent cause of death $>5$ years after diagnosis, secondary malignant tumours, cardiac disease and end-stage renal disease were also major causes of mortality.

The hazard ratios for hypertension (8.2), congestive heart failure (23.6) and renal failure (50.7) are all increased among 5-year survivors of WT compared with sibling groups ${ }^{15,185,186}$. The risk of congestive heart failure increases with the cumulative dose of doxorubicin administered, with a critical threshold of $240 \mathrm{mg} / \mathrm{m}^{2}$. Cardiotoxicity is potentiated by the concurrent use of radiotherapy, with girls and infants more susceptible than boys ${ }^{15,186}$. Similarly, doxorubicin seems to potentiate the adverse effects related to radiotherapy, probably owing to its radiosensitization of cells. These adverse effects include abnormal tissue growth within the target area and secondary malignancies.

The 20-year cumulative incidence of end-stage renal disease is reported to be $<1 \%$ in patients with unilateral $\mathrm{WT}$ and $\sim 10 \%$ in patients with bilateral disease ${ }^{187-190}$. The risk factors associated with end-stage renal disease owing to chronic renal failure are stromal-predominant histology (HR 6.4), intralobar nephrogenic rests (HR 5.9), and age at diagnosis of $<24$ months or $<48$ months (HR 1.7 and 2.8, respectively $)^{191}$. Given the increased risk of cardiovascular morbidity with chronic kidney disease, identifying patients with a high risk of progressive renal impairment early is imperative to preserve the quality of life of long-term survivors. The wider availability 
Oophorectomy

A surgical procedure to remove one or both ovaries. and accuracy of patient genotyping may identify more molecular fingerprints with implications for renal function into adulthood, in order to select a subset of patients with WT without clinical renal impairment at presentation, yet who might benefit from nephron-sparing surgical procedures.

Gonadal dysfunction. Gonadal dysfunction is observed in female WT survivors ${ }^{192,193}$, and is strongly associated with exposure of the ovaries to radiotherapy (at any dose) and treatment with alkylating drugs ${ }^{192,194}$. The first-line chemotherapy with two drugs used (that is, vincristine and actinomycin D), in general, does not affect either ovarian reserve or male fertility. Wholeabdomen radiation usually results in primary ovarian failure or premature menopause. Additionally, exposure of patients with WT to treatment including anthracyclines and lung radiation is associated with cardiovascular risks that can affect pregnancy outcomes ${ }^{192}$. WT survivors should receive personalized counselling about the type and magnitude of reproductive health risks on the basis of their specific treatment exposure, with older girls with unfavourable histology or high-risk WT being at increased risk. Patients at the highest risk should be offered fertility preservation whenever possible, and after accurate counselling ${ }^{195}$. In this view, prior abdominopelvic surgery (done to perform nephrectomy as part of initial cancer treatment) should not be regarded as a barrier to laparoscopic oophorectomy with tissue cryopreservation for fertility preservation.

\section{Quality of life}

Parents, charities and survivors of WT have worked closely with researchers and scientists to ensure that research is focused on what is important to families, and to highlight areas of need (BOX 3). The Wilms Tumour Link Group is an example of a parent-led research group

\section{Box 3 | Patient experience}

The statement provided has not been edited and the patient's emphases remain in place. Teenage years, the best ones in everyone's life. I was living unforgettable moments, going out and having parties with my friends. And then, after some medical checks, hell overnight. I had cancer. At first, I started imagining what I would have had to go through, how much I would have suffered. I was lost in doubts, fear and contrasting feelings.

To start chemotherapy shocked me. Eight hours, each impressed in my mind, in which milliards of medicine's drops came into my body. I felt exhausted.

As I was left alone for a moment in that hospital room, I abandoned myself to tears at the idea of repeating all of that the next day, and for eight more courses: that thought killed me. I came in that realization in that right moment.

I remember how important it was, for me, to have my friends around and to spend as much time as possible together. I remember they were the only ones who made me laugh, who made me feel normal, like nothing had ever changed. They made me breath, giving me the oxygen I needed. They reminded me how strong I was, when I was totally worn out. They recalled me what it meant to live, as sometimes I forgot how to do it.

Then, the COVID-19 pandemic situation came, and loneliness. My mum and I, stop. Far away from everyone, from everything. Three months of physical pain for chemo that I kept doing, of discouragement and fear. And, if it wasn't enough, there was also the worry of catching the virus.

Finally, after never-ending months, I came back to my lovely Naples. To my friends, to my family. To the sea, as I saw it, I felt free. Everything finally came to an end, and I couldn't believe it.

-G.B., 16 years old. in the UK focused on identifying priorities for future research, and uses a social media group of $>600$ members from around the world to communicate research updates. Parents of children with WT have participated and presented at international scientific meetings and are considered to be partners in the research process, amplifying the patient voice within childhood cancer research ${ }^{90}$.

This level of parent and patient involvement in research provides an important opportunity for physicians to work collaboratively. This collaboration facilitates the chance to have a greater impact on what is researched and highlights that not only is cure important but also the child's quality of life and happiness and health after cancer. The active involvement of parents and survivors in research helps translate findings in an equitable and accessible way. Findings are all too often kept within scientific journals that do not allow access to non-academics, so those that are affected by the disease are less informed. Working collaboratively and honestly with families is the key to patient-driven research with real-life translatable outcomes.

As a result of their better quality of life and physical functioning, children surviving renal tumours can hopefully also enjoy an increased involvement in sports. Patient counselling should include explaining any potential contraindication for practising sports carrying a risk of abdominal injuries ${ }^{196}$. Practice guidelines, where available, addressing the participation of children and adolescents with a solitary kidney (like most survivors of WT) in high-impact sports do not share a common vision worldwide ${ }^{197}$. In Italy, unlike the rest of Europe and the $\mathrm{USA}^{198}$, having only one kidney automatically disqualifies an individual wishing to participate in any organized competitive contact sport, including basketball and soccer, and sometimes, volleyball (BOX 4).

To instigate positive changes in cancer care through exercise, and to endorse change in patients sensitively, patients, families and health-care teams must be made more aware of current evidence-based information to provide a framework for the harmonization of guidelines for sport participation of renal tumour survivors, to ensure that they can exercise freely yet safely.

\section{Outlook}

\section{Basic research}

Increased understanding of the aberrant molecular pathways active in Wilms tumorigenesis has identified many potential targeted therapeutic approaches that could be applied in a clinical setting ${ }^{36}$. These include miRNA therapeutic modulation ${ }^{43,44}$, Wnt signalling ${ }^{90}$ and p53-specific biological targeting agents in anaplastic $\mathrm{WTs}^{69,70}$. In addition, retinoic acid, although ineffective as a WT therapy in the all-trans form ${ }^{199}$, may impart a differentiation effect on precancerous nephrotic rests in the 13-cis form, potentially mitigating the development of WT in a selected group of patients at risk, particularly those with hyperplastic nephroblastomatosis ${ }^{200,201}$. Although the options seem intriguing, the main challenges are the relatively few patients in each molecular subgroup, WT intratumoural heterogeneity ${ }^{78}$, few actionable known targets, selection and conduct of 
Box 4 | International controversies in advice on sporting activities in people with single kidneys

- Most children diagnosed with Wilms tumour (WT) become long-term survivors and living with a surgically solitary kidney.

- Among injuries occurring during sport exposure, the incidence of injuries to the kidneys is very low (sporting kidney injuries are $0.07-0.5 \%$ of all sports-related injuries), less frequent than head injuries, and usually without serious sequelae ${ }^{23,236}$.

- The recommendations for the participation of children and adolescents with a solitary kidney in contact or collision sports have changed over time. The last update from the American Academy of Paediatrics (2001) leans towards player participation without restriction in non-contact sports, and with individual assessment for limited-contact, contact, and collision sports to arrive at an unbiased judgement, which is not based only on the fact of having a solitary kidney ${ }^{198}$.

- However, national advice towards permission to participate in high-impact sports varies between countries and over time ${ }^{191}$

- Flank protectors have not been rigorously evaluated and an international standard for the protection they may offer is not available ${ }^{196}$.

- Individual counselling and decision-making between child, families and oncologist are recommended. for the detection and quantification of lung nodules (and disease elsewhere), which dictates intensity of treatment. The import of such technology is magnified by the shift from chest plain radiography to adequate CT scans, which provide detailed information on lung lesions, but also reveal that there is considerable inter-rater and intra-rater variation in interpreting such lesions even by experienced radiologists ${ }^{206}$.

WT may be amenable to advances in liquid biopsy techniques for diagnostics, monitoring of therapy, and detection of minimal residual disease ${ }^{80,118,207}$. As an example, COG study AREN1921 (NCT04322318) is collecting serial blood and urine samples in newly diagnosed anaplastic WT and patients with relapsed favourable histology WT to test the potential benefits of liquid biopsy ${ }^{208}$.

\section{Cooperative group efforts}

Both the COG and the SIOP groups have advanced well-organized prospective clinical trials and studies that tightly integrate biological aims and clinical insights, both linked to specific clinical protocols (AREN1921; NCT04322318) or via overarching biobanking and risk-stratification studies (AREN03B2;NCT00898365, SIOP UMBRELLA ${ }^{10}$. Cross-validation (meta-analysis) of data between these groups, especially data from small cohorts of rare patients (such as those with anaplastic tumours, bilateral tumours or relapsing disease) and strategic efforts to synergize intervention trials or observational studies hold promise in continuing to advance diagnostics, risk stratification and therapeutic options. Such 'harmonization' between cooperative groups has been formally advanced in the form of the Harmonica Initiative that integrates multidisciplinary dialogue, meetings, consensus building, specific research focus and overall strategies on a trans-continental, inter-cooperative group level ${ }^{6,90,127,202}$. Likewise, dialogue continues regarding potential trans-Atlantic collaborative trials among the Innovative Therapies for Children with Cancer (ITCC), the Pediatric Early Phase Clinical Trials Network (PEP-CTN), the Pediatric Preclinical Testing Consortium, and parent representatives ${ }^{90}$.

in refined personalized multilayer logically derived WT treatment will emerge, in the shorter term, via expanded creation and use of tumour models, ideally sufficient in number to represent the majority of WT biological subtypes ${ }^{86,87}$. Development of organoids, spheroids and PDX models has progressed from basic investigation to real-time patient-specific drug screening, and application of these approaches is now feasible, with plans evolving to launch an international patient-individualized relapse WT protocol harnessing this opportunity ${ }^{90,204}$.

Novel imaging investigations also hold promise for advancing WT treatment. For example, diffusionweighted imaging MRI has been implemented as standard for diagnostic and post-chemotherapy assessment ${ }^{105}$. Such techniques may non-invasively quantify tumours and risk-stratify patients with WT prior to surgery, with radiological surrogates (apparent diffusion coefficient mapping) for both necrosis (particularly relevant when tumour size remains stable) and blastemal-type histology ${ }^{205}$. Radiogenomics holds promise to further expand the utility of imaging in the care of patients with WT, as do artificial learning algorithms, for example,

\section{Global efforts}

Childhood cancer therapy in LMIC lags behind in diagnosis, therapy and survival, with minimal clinical or biological research. In 2018, WHO launched the Global Initiative for Childhood Cancer. In 2020, WHO published the CureAll Framework technical package WHO Global Initiative for Childhood Cancer, designed to provide guidance to member states for the implementation of childhood cancer services in resource-constrained settings ${ }^{174}$. Six tracer cancers, including WT, are targeted to provide guidance for diagnosis, therapy and supportive and survivorship care. With the help of international paediatric cancer partners (academic, regional and global societies and non-governmental organizations), the aim is to establish the necessary training and design of basic, and translational and clinical research ${ }^{174}$. Hence, progress in WT survival rates is expected to become more visible in the current decade.

Published online: 14 October 2021 
1. Pastore, G. et al. Malignant renal tumours incidence and survival in European children (1978-1997): report from the Automated Childhood Cancer Information System project. Eur. J. Cancer 42, 2103-2114 (2006)

2. Nakata, K., Colombet, M., Stiller, C. A. Pritchard-Jones, K. \& Steliarova-Foucher, E. Incidence of childhood renal tumours: an international population-based study. Int. J. Cancer 147 , 3313-3327 (2020)

3. Treger, T. D., Chowdhury, T., Pritchard-Jones, K. $\Sigma$ Behjati, S. The genetic changes of Wilms tumour. Nat. Rev. Nephrol. 15, 240-251 (2019).

4. Young, M. D. et al. Single-cell transcriptomes from human kidneys reveal the cellular identity of renal tumors. Science 361, 594-599 (2018).

5. Coorens, T. H. H. et al. Embryonal precursors of Wilms tumor. Science 366, 1247-1251 (2019). Comprehensive phylogenetic analysis that found premalignant clonal expansions (defined by somatic mutations shared between tumour and normal tissues but absent from blood cells) in morphologically normal kidney that preceded WT development. Clonal expansions evolving before the divergence of left and right kidney primordia may explain a proportion of bilateral WT cases.

6. Dome, J. S. et al. Advances in Wilms tumor treatment and biology: progress through international collaboration. J. Clin. Oncol. 33, 2999-3007 (2015). Pritchard-Jones, K. et al. Omission of doxorubicin from the treatment of stage II-III, intermediate-risk Wilms' tumour (SIOP WT 2001): an open-label, non-inferiority, randomised controlled trial. Lancet 386, 1156-1164 (2015).

This trial is the first to demonstrate in a series of $>500$ patients that doxorubicin can be safely omitted in most patients with stage III WT when classified as postoperative SIOP intermediate risk

8. Graf, N., Tournade, M. F. \& de Kraker, J. The role of preoperative chemotherapy in the management of Wilms' tumor. The SIOP studies. International Society of Pediatric Oncology. Urol. Clin. North. Am. 27, 443-454 (2000).

9. Vujanić, G. M. et al. The UMBRELLA SIOP-RTSG 2016 Wilms tumour pathology and molecular biology protocol. Nat. Rev. Urol. 15, 693-701 (2018).

This consensus paper describes the most up-to-date staging and histological classifications of WT according to SIOP

10. Van Den Heuvel-Eibrink, M. M. et al. Position paper rationale for the treatment of Wilms tumour in the UMBRELLA SIOP-RTSG 2016 protocol. Nat. Rev. Urol. 14, 743-752 (2017)

11. Dome, J. S. et al. Children's Oncology Group's 2013 blueprint for research: renal tumors. Pediatr. Blood Cancer 60, 994-1000 (2013).

12. Neuzil, K. et al. Health disparities among Tennessee pediatric renal tumor patients. J. Pediatr. Surg. 55, 1081-1087 (2020)

13. Gatta, G. et al. Childhood cancer survival in Europe 1999-2007: results of EUROCARE-5-a populationbased study. Lancet Oncol. 15, 35-47 (2014).

14. Cunningham, M. E. et al. Global disparities in Wilms tumor. J. Surg. Res. 247, 34-51 (2020).

15. Termuhlen, A. M. et al. Twenty-five year follow-up of childhood Wilms tumor: a report from the Childhood Cancer Survivor Study. Pediatr. Blood Cancer 57. 1210-1216 (2011)

16. Suh, E. et al. Late mortality and chronic health conditions in long-term survivors of early-adolescent and young adult cancers: a retrospective cohort analysis from the Childhood Cancer Survivor Study. Lancet Oncol. 21, 421-435 (2020)

17. Waters, A. M. \& Pritchard-Jones, K. Paediatrics: Long-term effects of Wilms tumour therapy on renal function. Nat. Rev. Urol. 12, 423-424 (2015).

18. Mifsud, W. \& Pritchard-Jones, K. Paediatrics: integrating genomics to dig deeper into Wilms tumour biology. Nat. Rev. Urol. 14, 703-704 (2017).

19. Steliarova-Foucher, E. et al. International incidence of childhood cancer, 2001-10: a population-based registry study. Lancet Oncol. 18, 719-731 (2017).

20. Ferlay J. et al. Global Cancer Observatory: Cancer Today. International Agency for Research on Cancer https://gco.iarc.fr/tomorrow (2021).

21. Stiller, C. A. \& Parkin, D. M. International variations in the incidence of childhood renal tumours. Br. J. Cancer 62, 1026-1030 (1990).

22. Bhakta, N. et al. Childhood cancer burden: a review of global estimates. Lancet Oncol. 20, e42-e53 (2019). A very comprehensive analysis on (challenging) estimates of the childhood global cancer burden, also proposing recommendations to strengthen data collection and improve and standardize analyses.

23. Ward, Z. J., Yeh, J. M., Bhakta, N., Frazier, A. L. \& Atun, R. Estimating the total incidence of global childhood cancer: a simulation-based analysis. Lancet Oncol. 20, 483-493 (2019).

24. Parkin, D. M. et al. Stage at diagnosis and survival by stage for the leading childhood cancers in three populations of sub-Saharan Africa. Int. J. Cancer 148 , 2685-2691 (2021).

25. Merks, J. H. M. Caron, H. N.\& Hennekam, R. C. M. High incidence of malformation syndromes in a series of 1,073 children with cancer. Am. J. Med. Genet. 134 A, 132-143 (2005).

26. Scott, R. H., Stiller, C. A., Walker, L. \& Rahman, N Syndromes and constitutional chromosomal abnormalities associated with Wilms tumour. J. Med. Genet. 43, 705-715 (2006)

27. Little J., Epidemiology of childhood cancer. IARC Scientific Publication N149 (IARC, 1999).

28. Brioude, F. et al. Expert consensus document: clinical and molecular diagnosis, screening and management of Beckwith-Wiedemann syndrome: an international consensus statement. Nat. Rev. Endocrinol. 4, 229-249 (2018)

29. Hol, J. A et al. Wilms tumour surveillance in at-risk children: literature review and recommendations from the SIOP-Europe Host Genome Working Group and SIOP Renal Tumour Study Group. Eur. J. Cancer 153, 51-63 (2021).

This study reports on updated WT surveillance guidelines for children with genetic risk of developing WT.

30. Breslow, N. E. et al. Characteristics and outcomes of children with the Wilms tumor-aniridia syndrome a report from the National Wilms Tumor Study Group. J. Clin. Oncol. 21, 4579-4585 (2003).

31. Brok, J., Treger, T. D., Gooskens, S. L., van den Heuvel-Eibrink, M. M. \& Pritchard-Jones, K Biology and treatment of renal tumours in childhood. Eur. J. Cancer 68, 179-195 (2016).

32. Charlton, J., Irtan, S., Bergeron, C. \& Pritchard-Jones, K Bilateral Wilms tumour: a review of clinical and molecular features. Expert. Rev. Mol. Med. 19, e8 (2017).

33. Nakata, K. et al. Comparative analysis of the clinical characteristics and outcomes of patients with Wilms tumor in the United Kingdom and Japan. Pediatr. Blood Cancer 68, e29143 (2021).

34. Fukuzawa, R. et al. Epigenetic differences between Wilms' tumours in white and east-Asian children. Lancet 363, 446-451 (2004).

35. Breslow, N. E., Beckwith, J. B., Perlman, E. J. $\&$ Reeve, A. E. Age distributions, birth weights, nephrogenic rests, and heterogeneity in the pathogenesis of Wilms tumor. Pediatr. Blood Cancer 47, 260-267 (2006)

36. Behjati, S., Gilbertson, R. J. \& Pfister, S. M. Maturation block in childhood cancer. Cancer Discov. 11, 542-544 (2021)

37. McMahon, A. P. Development of the mammalian kidney. Curr. Top. Dev. Biol. 117, 31-64 (2016).

38. Huff, V. Wilms' tumours: about tumour suppressor genes, an oncogene and a chameleon gene. Nat. Rev. Cancer 11, 111-121 (2011).

39. Grundy, P. E. et al. Loss of heterozygosity for chromosomes $1 p$ and $16 q$ is an adverse prognostic factor in favorable-histology Wilms tumor: a report from the National Wilms Tumor Study Group. J. Clin Oncol. 23, 7312-7321 (2005)

This trial for the first time integrated molecular prognostic markers into WT risk and treatment classification.

40. Gratias, E. J. et al. Association of chromosome 1q gain with inferior survival in favorable-histology Wilms tumor: a report from the Children's Oncology Group. J. Clin. Oncol. 34, 3189-3194 (2016).

41. Chagtai, T. et al. Gain of 1q as a prognostic biomarker in Wilms tumors (WTs) treated with preoperative chemotherapy in the International Society of Paediatric Oncology (SIOP) WT 2001 trial: a SIOP Renal Tumours Biology Consortium study. J. Clin. Oncol. 34, 3195-3203 (2016)

42. Gadd, S. et al. A Children's Oncology Group and TARGET initiative exploring the genetic landscape of Wilms tumor. Nat. Genet. 49, 1487-1494 (2017).

First comprehensive genome-wide sequencing, mRNA and miRNA expression, DNA copy number, and DNA methylation analysis in a series of 117 WTs, followed by targeted sequencing of 651 WTs, identifying mutations in genes not previously recognized as recurrently involved in WT.

43. Walz, A. L. et al. Recurrent DGCR8, DROSHA, and SIX homeodomain mutations in favorable histology Wilms tumors. Cancer Cell 27, 286-297 (2015).

44. Wegert, J. et al. Mutations in the SIX1/2 pathway and the DROSHA/DGCR8 miRNA microprocessor complex underlie high-risk blastemal type Wilms tumors. Cancer Cell 27, 298-311 (2015)

45. Call, K. M. et al. Isolation and characterization of a zinc finger polypeptide gene at the human chromosome 11 Wilms' tumor locus. Cell 60 509-520 (1990)

46. Gessler, M. et al. Homozygous deletion in Wilms tumours of a zinc-finger gene identified by chromosome jumping. Nature 343, 774-778 (1990).

47. Schumacher, V. et al. Correlation of germ-line mutations and two-hit inactivation of the WT1 gene with Wilms tumors of stromal-predominant histology. Proc. Natl Acad. Sci. USA 94, 3972-3977 (1997).

48. Pelletier, J. et al. Germline mutations in the Wilms' tumor suppressor gene are associated with abnormal urogenital development in Denys-Drash syndrome. Cell 67, 437-447 (1991)

49. Barbaux, S. et al. Donor splice-site mutations in WT1 are responsible for Frasier syndrome. Nat. Genet. 17 467-470 (1997).

50. Klamt, B. et al. Frasier syndrome is caused by defective alternative splicing of WT 1 leading to an altered ratio of WT1 +/- KTS splice isoforms Hum. Mol. Genet. 7, 709-714 (1998).

51. Koesters, R. et al. Mutational activation of the beta-catenin proto-oncogene is a common event in the development of Wilms' tumors. Cancer Res. 16 3880-3882 (1999).

52. Scott, R. H. et al. Stratification of Wilms tumor by genetic and epigenetic analysis. Oncotarget 3 327-335 (2012)

53. Kaneko, Y. et al. A high incidence of WT1 abnormality in bilateral Wilms tumours in Japan, and the penetrance rates in children with WT1 germline mutation. Br. J. Cancer 112, 1121-1133 (2015).

54. Wegert, J. et al. WTX inactivation is a frequent, but late event in Wilms tumors without apparent clinical impact. Genes. Chromosom. Cancer 48, 1102-1111 (2009).

55. Rakheja, D. et al. Somatic mutations in DROSHA and DICER1 impair microRNA biogenesis through distinct mechanisms in Wilms tumours. Nat. Commun. 2, 4802 (2014).

56. Torrezan, G. T. et al. Recurrent somatic mutation in DROSHA induces microRNA profile changes in Wilms tumour. Nat. Commun. 5, 4039 (2014).

57. Wu, M. K. et al. Evolution of renal cysts to anaplastic sarcoma of lidney in a child with DICER 1 syndrome. Pediatr. Blood Cancer 63, 1272-1275 (2016).

58. Hill, D. A et al. DICER1 mutations in familial pleuropulmonary blastoma. Science 325, 965 (2009).

59. Williams, R. D. et al. Multiple mechanisms of MYCN dysregulation in Wilms tumour. Oncotarget 6 , 7232-7243 (2015).

60. Williams, R. D. et al. Subtype-specific FBXW7 mutation and MYCN copy number gain in Wilms' tumor. Clin. Cancer Res. 16, 2036-2045 (2010).

61. Xu, J. et al. Eya 1 interacts with Six 2 and Myc to regulate expansion of the nephron progenitor pool during nephrogenesis. Dev. Cell 31, 434-447 (2014).

62. Hanks, S. et al. Germline mutations in the PAF complex gene CTR9 predispose to Wilms tumour Nat. Commun. 5, 4398 (2014).

63. Hol, J. A. et al. TRIM28 variants and Wilms' tumour predisposition. J. Pathol. 254, 494-504 (2021).

64. Diets, I. J. et al. TRIM28 haploinsufficiency predisposes to Wilms tumor. Int. J. Cancer 145 , 941-951 (2019)

65. Armstrong. A. E. et al. A unique subset of low-risk Wilms tumors is characterized by loss of function of TRIM28 (KAP1), a gene critical in early renal development: a Children's Oncology Group study. PLOS ONE 13, e0208936 (2018).

66. Halliday, B. J. et al. Germline mutations and somatic inactivation of TRIM28 in Wilms tumour. PLoS Genet. 14, e1007399 (2018).

67. Kenny, C. et al. Mutually exclusive BCOR internal tandem duplications and YWHAE-NUTM2 fusions in clear cell sarcoma of kidney: not the full story. J. Pathol. 238, 617-620 (2016)

68. Ueno-Yokohata, H. et al. Consistent in-frame internal tandem duplications of BCOR characterize clear cell sarcoma of the kidney. Nat. Genet. 47, 861-863 (2015). 
69. Maschietto, M. et al. TP53 mutational status is a potential marker for risk stratification in Wilms tumour with diffuse anaplasia. PLoS ONE 9, e109924 (2014).

70. Ooms, A. H. A. G. et al. Significance of TP53 mutation in Wilms tumors with diffuse anaplasia: a report from the Children's Oncology Group. Clin. Cancer Res. 22 5582-5591 (2016).

71. Wegert, J. et al. TP53 alterations in Wilms tumour represent progression events with strong intratumour heterogeneity that are closely linked but not limited to anaplasia. J. Pathol. Clin. Res. 3, 234-248 (2017).

72. Maciaszek, J. L., Oak, N. \& Nichols, K. E. Recent advances in Wilms' tumor predisposition. Hum. Mol. Genet. 29, R138-R149 (2020).

73. Mahamdallie, S. et al. Identification of new Wilms tumour predisposition genes: an exome sequencing study. Lancet Child. Adolesc. Heal. 3, 322-331 (2019).

74. Beckwith, J. B., Kiviat, N. B. \& Bonadio, J. F. Nephrogenic rests, nephroblastomatosis, and the pathogenesis of Wilms' tumor. Fetal Pediatr. Pathol. 10, 1-36 (1990)

75. Vujanić, G. M. et al. Nephrogenic rests in Wilms tumors treated with preoperative chemotherapy: the UK SIOP Wilms Tumor 2001 Trial experience. Pediatr. Blood Cancer 64, e26547 (2017).

76. Fukuzawa, R., Heathcott, R. W., More, H. E. \& Reeve, A. E. Sequential WT1 and CTNNB1 mutations and alterations of $\beta$-catenin localisation in intralobar nephrogenic rests and associated Wilms tumours: two case studies. J. Clin. Pathol. 60, 1013-1016 (2007).

77. Vuononvirta, R. et al. Perilobar nephrogenic rests are nonobligate molecular genetic precursor lesions of insulin-like growth factor-II-associated Wilms tumors. Clin. Cancer Res. 14, 7635-7644 (2008).

78. Cresswell, G. D. et al. Intra-tumor genetic heterogeneity in Wilms tumor: clonal evolution and clinical implications. EBioMedicine 9, 120-129 (2016)

79. Van Paemel, R. et al. Minimally invasive classification of paediatric solid tumours using reduced representation bisulphite sequencing of cell-free DNA a proof-of-principle study. Epigenetics 16, 196-208 (2020).

80. Jiménez, I. et al. Circulating tumor DNA analysis enables molecular characterization of pediatric renal tumors at diagnosis. Int. J. Cancer 144, 68-79 (2019).

81. Hu, Q. et al. Wt1 ablation and Igf2 upregulation in mice result in Wilms tumors with elevated ERK $1 / 2$ phosphorylation. J. Clin. Invest. 121, 174-183 (2011).

82. Hunter, R. W. et al. Loss of Dis312 partially phenocopies Perlman syndrome in mice and results in upregulation of Igf2 in nephron progenitor cells. Genes Dev. 32, 903-908 (2018)

83. Urbach, A. et al. Lin28 sustains early renal progenitors and induces Wilms tumor. Genes Dev. 28, 971-982 (2014).

84. Moisan, A. et al. The WTX tumor suppressor regulates mesenchymal progenitor cell fate specification. Dev. Cell 20, 583-596 (2011).

85. Kruber, P. et al. Loss or oncogenic mutation of DROSHA impairs kidney development and function, but is not sufficient for Wilms tumor formation. Int. $J$. Cancer 144, 1391-1400 (2019).

86. Murphy, A. J. et al. Forty-five patient-derived xenografts capture the clinical and biological heterogeneity of Wilms tumor. Nat. Commun. 10, 5806 (2019)

87. Calandrini, C. et al. An organoid biobank for childhood kidney cancers that captures disease and tissue heterogeneity Nat Commun 11, 1310 (2020).

88. Wegert, J. et al. High-risk blastemal Wilms tumor can be modeled by 3D spheroid cultures in vitro. Oncogene 39, 849-861 (2020).

89. Schutgens, F. et al. Tubuloids derived from human adult kidney and urine for personalized disease modeling. Nat. Biotechnol. 37, 303-313 (2019)

90. Brok, J. et al. Unmet needs for relapsed or refractory Wilms tumour: mapping the molecular features, exploring organoids and designing early phase trials - a collaborative SIOP-RTSG, COG and ITCC session at the first SIOPE meeting. Eur. J. Cancer 144 113-122 (2021)

91. Mullen, E. \& Graf, N. in Renal tumors of childhood: biology and therapy 1 st edn (eds Pritchard-Jones, K. \& Dome, J. S.) 39-52 (Springer, 2014).

92. Fernandez, C. et al. in Pizzo \& Poplack's Pediatric Oncology 8th Edn Ch. 24 (eds Blaney, S. M., Helman, L. J. \& Adamson, P. C.) 956-972 (Wolters Kluwer Health, 2020).
93. Scott, R. H. et al. Surveillance for Wilms tumour in at-risk children: pragmatic recommendations for best practice. Arch. Dis. Child. 91, 995-999 (2006).

94. Wilde, J. C. H. et al. Challenges and outcome of Wilms tumour management in a resource-constrained setting. Afr. J. Paediatr. Surg. 7, 159-162 (2010).

95. Israels, T., Harif, M. \& Pritchard-Jones, K. Treatment of Wilms tumor in low-income countries: challenges and potential solutions. Future Oncol. 9, 1057-1059 (2013).

96. Vasquez, L. et al. Factors associated with the latency to diagnosis of childhood cancer in Peru. Pediatr. Blood Cancer 63, 1959-1965 (2016).

97. Ooms, A. H. A. G. et al. Renal tumors of childhooda histopathologic pattern-based diagnostic approach. Cancers 12, 729 (2020)

98. Beckwith, J. B. \& Palmer, N. F. Histopathology and prognosis of Wilms tumor: results from the first National Wilms' Tumor study. Cancer 41, 1937-1948 (1978).

99. Faria, P. et al. Focal versus diffuse anaplasia in Wilms tumor-new definitions with prognostic significance: a report from the National Wilms Tumor Study Group. Am. J. Surg. Pathol. 20, 909-920 (1996).

100. Dome, J. S. et al. Treatment of anaplastic histology Wilms' tumor: results from the fifth National Wilms' Tumor Study. J. Clin. Oncol. 24, 2352-2358 (2006).

101. Perlman, E. J. Pediatric renal tumors: practical updates for the pathologist. Pediatr. Dev. Pathol. 8 320-338 (2005)

102. Fernandez, C. V. et al. Outcome and prognostic factors in stage III favorable-histology Wilms tumor: a report from the Children's Oncology Group Study AREN0532. J. Clin. Oncol. 36, 254-261 (2018)

103. Kaste, S. C. et al. Wilms tumour: prognostic factors, staging, therapy and late effects. Pediatr. Radiol. 38, 2-17 (2008)

104. Israels, T. et al. SIOP PODC: clinical guidelines for the management of children with Wilms tumour in a low income setting. Pediatr. Blood Cancer 60, 5-11 (2013).

105. Watson, T., Oostveen, M., Rogers, H., Pritchard-Jones, K. \& Olsen, $\varnothing$. The role of imaging in the initial investigation of paediatric renal tumours. Lancet Child. Adolesc. Health 4, 232-241 (2020).

106. Sandberg, J. K. et al. Imaging characteristics of nephrogenic rests versus small Wilms tumors: a report from the Children's Oncology Group Study AREN03B2. Am. J. Roentgenol. 214, 987-994 (2020).

107. Khanna, G. et al. Detection of preoperative Wilms tumor rupture with CT: a report from the Children's Oncology Group. Radiology 266, 610-617 (2013).

108. Smets, A. M. J. B. et al. The contribution of chest CT-scan at diagnosis in children with unilateral Wilms' tumour. Results of the SIOP 2001 study. Eur. J. Cancer 48, 1060-1065 (2012).

109. Dix, D. B. et al. Treatment of stage IV favorable histology Wilms tumor with lung metastases: a report from the Children's Oncology Group AREN0533 study. J. Clin. Oncol. 36, 1564-1570 (2018).

110. Littooij, A. S. et al. Apparent diffusion coefficient as it relates to histopathology findings in postchemotherapy nephroblastoma: a feasibility study. Pediatr. Radiol. 47, 1608-1614 (2017)

111. Iaboni, D. S. M., Chi, Y. Y., Kim, Y., Dome, J. S. \& Fernandez, C. V. Outcome of Wilms tumor patients with bone metastasis enrolled on National Wilms Tumor Studies 1-5: a report from the Children's Oncology Group. Pediatr. Blood Cancer 66, e27430 (2019).

112. Seibel, N. L. et al. Impact of cyclophosphamide and etoposide on outcome of clear cell sarcoma of the kidney treated on the National Wilms Tumor Study-5 (NWTS-5). Pediatr. Blood Cancer 66, e27450 (2019).

113. Brok, J. et al. Relapse of Wilms' tumour and detection methods: a retrospective analysis of the 2001 Renal Tumour Study Group-International Society of Paediatric Oncology Wilms' tumour protocol database. Lancet Oncol. 19, 1072-1081 (2018)

First detailed analysis in a series of $>4,000$ patients on methods to detect WT relapse, laying the fundation for improved evidence-based follow-up schemes.

114. Charlebois, J., Rivard, G. E. \& St-Louis, J. Management of acquired von Willebrand syndrome. Transfus. Apher. Sci. 57, 721-723 (2018).

115. Jackson, T. J. et al. The diagnostic accuracy and clinical utility of pediatric renal tumor biopsy: repor of the UK experience in the SIOP UK WT 2001 trial. Pediatr Blood Cancer 66, e27627 (2019).
116. Brisse, H. J., de la Monneraye, Y., Cardoen, L. \& Schleiermacher, G. From Wilms to kidney tumors: which ones require a biopsy? Pediatr. Radiol. 50, 1049-1051 (2020).

117. Weiser, D. A. et al. Progress toward liquid biopsies in pediatric solid tumors. Cancer Metastasis Rev. 38 553-571 (2019)

118. Treger, T. D. et al. Somatic TP53 mutations are detectable in circulating tumor DNA from children with anaplastic wilms tumors. Transl Oncol. 11 1301-1306 (2018).

119. Groenendijk, A. et al. Prognostic factors for Wilms tumor recurrence: a review of the literature. Cancers 13, 3142 (2021)

120. Dome, J. S., Perlman, E. J. \& Graf, N. Risk stratification for Wilms tumor: current approach and future directions. Am. Soc. Clin. Oncol. Educ. B 34 215-223 (2014).

121. Nelson, M. V., van den Heuvel-Eibrink, M. M., Graf, N. \& Dome, J. S. New approaches to risk stratification for Wilms tumor. Curr. Opin. Pediatr. 33, 40-48 (2021).

122. Vujanić, G. M. et al. Revised International Society of Paediatric Oncology (SIOP) working classification of renal tumors of childhood. Med. Pediatr. Oncol. 38, 79-82 (2002).

123. Verschuur, A et al. Treatment of pulmonary metastases in children with stage IV nephroblastoma with risk-based use of pulmonary radiotherapy. J. Clin Oncol. 30, 3533-3539 (2012).

124. Van Den Heuvel-Eibrink, M. M. et al. Outcome of localised blastemal-type Wilms tumour patients treated according to intensified treatment in the SIOP WT 2001 protocol, a report of the SIOP Renal Tumour Study Group (SIOP-RTSG). Eur. J. Cancer 51, 498-506 (2015)

125. Daw, N. C. et al. Activity of vincristine and irinotecan in diffuse anaplastic Wilms tumor and therapy outcomes of stage II to IV disease: results of the Children's Oncology Group AREN0321 study. J. Clin. Oncol. 38 1558-1568 (2020)

126. Pasqualini, C. et al. Outcome of patients with stage IV high-risk Wilms tumour treated according to the SIOP2001 protocol: a report of the SIOP Renal Tumour Study Group. Eur. J. Cancer 128, 38-46 (2020).

127. Malogolowkin, M. H. et al. Incidence and outcomes of patients with late recurrence of Wilms' tumor. Pediatr. Blood Cancer 60, 1612-1615 (2013).

128. Mullen, E. A. et al. Impact of surveillance imaging modality on survival after recurrence in patients with favorable-histology Wilms tumor: a report from the Children's Oncology Group. J. Clin. Oncol. 36 3396-3403 (2018).

129. Spreafico, F. et al. Treatment of relapsed Wilms tumors: lessons learned. Expert. Rev. Anticancer. Ther 9, 1807-1815 (2009).

130. Spreafico, F. et al. High dose chemotherapy and autologous hematopoietic cell transplantation for Wilms tumor: a study of the European Society for Blood and Marrow Transplantation. Bone Marrow Transpl. 55, 376-383 (2020).

131. Kratz, C. P. et al. Predisposition to cancer in children and adolescents. Lancet Child. Adolesc. Health $\mathbf{5}$, 142-154 (2021)

132. Apple, A. \& Lovvorn, H. N. Wilms tumor in subSaharan Africa: molecular and social determinants of a global pediatric health disparity. Front. Oncol. 10 606380 (2020)

133. Fiala, E. M. et al. 11 p15.5 epimutations in children with Wilms tumor and hepatoblastoma detected in peripheral blood. Cancer 126, 3114-3121 (2020)

134. Godzinski, J., Graf, N. \& Audry, G. Current concepts in surgery for Wilms tumor-the risk and functionadapted strategy. Eur. J. Pediatr. Surg. 24, 457-460 (2014).

135. Lopyan, N. M. \& Ehrlich, P. F. Surgical management of Wilms tumor (nephroblastoma) and renal cell carcinoma in children and young adults. Surg. Oncol. Clin. N. Am. 30, 305323 (2021)

136. Green, D. M. et al. Treatment with nephrectomy only for small, stage l/favorable histology Wilms' tumor: a report from the National Wilms' Tumor Study Group. J. Clin. Oncol. 19, 3719-3724 (2001).

137. Ehrlich, P. et al. Results of the first prospective multi-institutional treatment study in children with bilateral Wilms tumor (AREN0534): a report from the Children's Oncology Group. Ann. Surg. 266, 470-478 (2017).

138. Ehrlich, P. F. et al. Results of treatment for patients with multicentric or bilaterally predisposed unilateral Wilms tumor (AREN0534): a report from the 
Children’s Oncology Group. Cancer 126, 3516-3525 (2020).

139. Shamberger, R. C. et al. Intravascular extension of Wilms tumor. Ann. Surg. 234, 116-121 (2001)

140. Ritchey, M. et al. Ureteral extension in Wilms' tumor: a report from the National Wilms' Tumor Study Group (NWTSG) J J. Pediatr. Surg. 43, 1625-1629 (2008).

141. Gow, K. W. et al. Primary nephrectomy and intraoperative tumor spill: report from the Children's Oncology Group (COG) renal tumors committee. J. Pediatr. Surg. 48, 34-38 (2013).

142. Ehrlich, P. F. et al. Surgical protocol violations in children with renal tumors provides an opportunity to improve pediatric cancer care: a report from the Children's Oncology Group. Pediatr. Blood Cancer 63, 1905-1910 (2016)

143. Aldrink, J. H. et al. Technical considerations for nephron-sparing surgery in children: what is needed to preserve renal units? J. Surg. Res. 232, 614-620 (2018)

144. Murphy, A. \& Davidoff, A. Bilateral Wilms tumor: a surgical perspective Children 5,134 (2018).

145. Cox, S., Büyükünal, C. \& Millar, A. J. W. Surgery for the complex Wilms tumour. Pediatr. Surg. Int. 36, 113-127 (2020)

146. Malek, M. M. et al. Minimally invasive surgery for pediatric renal tumors: a systematic review by the APSA Cancer Committee. J. Pediatr. Surg. 55 2251-2259 (2020)

147. Fernandez, C. V. et al. Clinical outcome and biological predictors of relapse after nephrectomy only for very low-risk Wilms tumor: a report from Children's Oncology Group AREN0532. Ann. Surg. 265, 835-840 (2017)

148. Green, D. M. The treatment of stages I-IV favorable histology Wilms' tumor. J. Clin. Oncol. 22, 1366-1372 (2004).

149. Green, D. M. The evolution of treatment for Wilms tumor. J. Pediatr. Surg. 48, 14-19 (2013).

150. Green, D. M. et al. Outcome of patients with stage II/ favorable histology wilms tumor with and without local tumor spill: a report from the National Wilms Tumor Study Group. Pediatr. Blood Cancer 61, 134-139 (2014).

151. Dix, D. B. et al. Augmentation of therapy for combined loss of heterozygosity $1 p$ and $16 q$ in favorable histology Wilms tumor: a Children's Oncology Group AREN0532 and AREN0533 study report. J. Clin. Oncol. 37, 2769-2777 (2019).

152. Green, D. M. et al. Treatment of Wilms tumor relapsing after initial treatment with vincristine and actinomycin D: a report from the National Wilms Tumor Study Group. Pediatr. Blood Cancer 48 493-499 (2007)

153. Malogolowkin, M. et al. Treatment of Wilms tumor relapsing after initial treatment with vincristine, actinomycin $D$, and doxorubicin. A report from the National Wilms Tumor Study Group. Pediatr. Blood Cancer 50, 236-241 (2008).

154. Ha, T. C. et al. An international strategy to determine the role of high dose therapy in recurrent Wilms tumour. Eur. J. Cancer 49, 194-210 (2013).

155. Dome, J. S. et al. Impact of the first generation of Children's Oncology Group clinical trials on clinical practice for Wilms tumor. J. Natl Compr. Cancer Netw. 19, 978-985 (2021)

156. Kalapurakal, J. A. et al. Intraoperative spillage of favorable histology Wilms tumor cells: influence of irradiation and chemotherapy regimens on abdominal recurrence. a report from the National Wilms Tumor Study Group. Int. J. Radiat. Oncol. Biol. Phys. 76 201-206 (2010).

157. Kalapurakal, J. A et al. Cardiac-sparing whole lung intensity modulated radiation therapy in children with Wilms tumor: final report on technique and abdominal field matching to maximize normal tissue protection. Pract. Radiat. Oncol. 9, e62-e73 (2019).

158. Kalapurakal, J. A. et al. Outcomes of children with favorable histology Wilms tumor and peritoneal implants treated in National Wilms Tumor Studies-4 and -5. Int. J. Radiat. Oncol. Biol. Phys. 77, 554-558 (2010).

159. National Comprehensive Cancer Network. Wilms Tumor (Nephroblastoma). NCCN https://www.nccn.org guidelines/guidelines-detail? category $=1$ \&id $=1500$ (2021).

160. Tournade, M. F. et al. Optimal duration of preoperative therapy in unilateral and nonmetastatic Wilms' tumor in children older than 6 months: Results of the Ninth International Society of Pediatric Oncology Wilms' Tumor Trial and Study. J. Clin. Oncol. 19, 488-500 (2001).
161. Fajardo, R. D. et al. Is radiotherapy required in firstline treatment of stage I diffuse anaplastic Wilms tumor? A report of SIOP-RTSG, AIEOP, JWiTS, and UKCCSG. Pediatr. Blood Cancer 67, e28039 (2020).

162. Janssens, G. O. et al. The SIOP-Renal Tumour Study Group consensus statement on flank target volume delineation for highly conformal radiotherapy. Lancet Child. Adolesc. Health 4, 846-852 (2020).

163. Abuidris, D. O. et al. Wilms tumour in Sudan. Pediatr. Blood Cancer 50, 1135-1137 (2008)

164. Israels, T. et al. Improved outcome at end of treatment in the collaborative Wilms tumour Africa project. Pediatr. Blood Cancer 65, e26945 (2018).

165. Valverde, P. et al. An analysis of treatment failure in Wilms tumor (WT): a report from the Central American Association of Pediatric Hematology/Oncology (AHOPCA) [abstract 57]. J. Glob. Oncol. 2 (Suppl. 3), 2s (2016).

166. Gibson, T. N. et al. Baseline characteristics and outcomes of children with cancer in the Englishspeaking Caribbean: a multinational retrospective cohort. Pediatr. Blood Cancer 65, e27298 (2018).

167. Lam, C. G., Howard, S. C., Bouffet, E. \& Pritchard-Jones, K. Science and health for all children with cancer. Science 363, 1182-1186 (2019).

168. Molyneux, E., Mathanga, D., Witte, D. \& Molyneux, M. Practical issues in relation to clinical trials in children in low-income countries: experience from the front line. Arch. Dis. Child. 97, 848-851 (2012)

169. Libes, J. et al. Risk factors for abandonment of Wilms tumor therapy in Kenya. Pediatr. Blood Cancer 62, 252-256 (2015)

170. Pribnow, A. K., Ortiz, R., Báez, L. F., Mendieta, L. \& Luna-Fineman, S. Effects of malnutrition on treatmentrelated morbidity and survival of children with cancer in Nicaragua. Pediatr. Blood Cancer 64, e26590 (2017).

171. Sala, A. et al. Nutritional status at diagnosis is related to clinical outcomes in children and adolescents with cancer: a perspective from Central America. Eur. J. Cancer 48, 243-252 (2012)

172. Israels, T. et al. Malnourished Malawian patients presenting with large Wilms tumours have a decreased vincristine clearance rate. Eur. J. Cancer 46, 1841-1847 (2010)

173. Israëls, T. et al. Acute malnutrition is common in Malawian patients with a Wilms tumour: a role for peanut butter. Pediatr. Blood Cancer 53, 1221-1226 (2009).

174. World Health Organization. WHO Global Initiative for Childhood Cancer: an Overview. WHO https://www. who.int/publications/m/item/global-initiative-forchildhood-cancer (2020)

175. Israels, T. et al. Management of children with a Wilms tumor in Malawi, sub-Saharan Africa. J. Pediatr. Hematol. Oncol. 34, 606-610 (2012).

176. Israels, T. et al. The efficacy and toxicity of SIOP preoperative chemotherapy in Malawian children with a Wilms tumour. Pediatr. Blood Cancer 59, 636-641 (2012).

177. Israëls, T. et al. Clinical trials to improve childhood cancer care and survival in sub-Saharan Africa. Nat. Rev. Clin. Oncol. 10, 599-604 (2013).

178. Chitsike, l. et al. Working together to build a better future for children with cancer in Africa. JCO Glob. Oncol. 6, 1076-1078 (2020).

179. Paintsil, V. et al. The Collaborative Wilms Tumour Africa Project: baseline evaluation of Wilms tumour treatment and outcome in eight institutes in subSaharan Africa. Eur. J. Cancer 51, 84-91 (2015).

180. Chagaluka, G. et al. Improvement of overall survival in the Collaborative Wilms Tumour Africa Project. Pediatr. Blood Cancer 67, e28383 (2020).

181. SIOP. Treatment Guidelines: Collaborative Wilms Tumour Africa Project. SIOP https://siop-online.org/ wp-content/uploads/2020/04/Treatment-GuidelinesCollaborative-Wilms-Tumour-Africa-Project-Phase-IIdoc-v1.8-FINAL.pdf (2020).

182. Oeffinger, K. C. et al. Chronic health conditions in adult survivors of childhood cancer. N. Engl. J. Med 355, 1572-1582 (2006)

183. Lee, J. S. et al. Second malignant neoplasms among children, adolescents and young adults with Wilms tumor. Pediatr. Blood Cancer 62, 1259-1264 (2015).

184. Cotton, C. A. et al. Early and late mortality after diagnosis of Wilms tumor. J. Clin. Oncol. 27 1304-1309 (2009).

185. Chu, D. I. et al. Kidney outcomes and hypertension in survivors of wilms tumor: a prospective cohort study. J. Pediatr. 230, 215-220 (2021).

186. Green, D. M. et al. Congestive heart failure after treatment for Wilms tumor: a report from the
National Wilms' Tumor Study Group. J. Clin. Oncol. 19, 1926-1934 (2001).

187. Green, D. M. et al. Long-term renal function after treatment for unilateral, nonsyndromic Wilms tumor. A report from the St. Jude Lifetime Cohort Study. Pediatr. Blood Cancer 67, e28271 (2020).

188. Breslow, N. E. et al. End stage renal disease in patients with Wilms tumor: results from the National Wilms Tumor Study Group and the United States Renal Data System. J. Urol. 174, 1972-1975 (2005).

189. Grigoriev, Y. et al. Treatments and outcomes for endstage renal disease following Wilms tumor. Pediatr. Nephrol. 27, 1325-1333 (2012).

190. Interiano, R. B. et al. Renal function in survivors of nonsyndromic Wilms tumor treated with unilateral radical nephrectomy. Cancer 121, 2449-2456 (2015).

191. Lange, J. et al. Risk factors for end stage renal disease in non-WT1-syndromic Wilms tumor. J. Urol. 186, 378-386 (2011).

192. Van Dorp, W. et al. Reproductive function and outcomes in female survivors of childhood, adolescent, and young adult cancer: a review. J. Clin. Oncol. 36 , 2169-2180 (2018).

193. Levitt, G. Renal tumours: long-term outcome. Pediatr. Nephrol. 27, 911-916 (2012)

194. Chemaitilly, W. et al. Premature ovarian insufficiency in childhood cancer survivors: a report from the St. Jude Lifetime Cohort. J. Clin. Endocrinol. Metab. 102, 2242-2250 (2017).

195. van den Berg, M. et al. Fertility among female survivors of childhood, adolescent, and young adult cancer: protocol for two pan-European studies (PanCareLIFE). JMIR Res. Protoc. 7, E10824 (2018).

196. Papagiannopoulos, D. \& Gong, E. Revisiting sports precautions in children with solitary kidneys and congenital anomalies of the kidney and urinary tract. Urology 101, 9-14 (2017).

197. Spreafico, F. et al. Why should survivors of childhood renal tumor and others with only one kidney be denied the chance to play contact sports? Expert. Rev. Anticancer. Ther. 14, 363-366 (2014).

198. Committee on Sports Medicine and Fitness. American Academy of Pediatrics: medical conditions affecting sports participation. Pediatrics 107, 1205-1209 (2001).

199. Adamson, P. C. et al. A phase 2 trial of all-transretinoic acid in combination with interferon- $\alpha 2 a$ in children with recurrent neuroblastoma or Wilms tumor: A Pediatric Oncology Branch, $\mathrm{NCl}$ and Children's Oncology Group Study. Pediatr. Blood Cancer 49, 661-665 (2007).

200. Friesenbichler, W. et al. Outcome of two patients with bilateral nephroblastomatosis/Wilms tumour treated with an add-on 13-cis retinoic acid therapy-case report. Pediatr. Hematol. Oncol. 35, 218-224 (2018).

201. Wegert, J. et al. Retinoic acid pathway activity in Wilms tumors and characterization of biological responses in vitro. Mol. Cancer 10, 136 (2011).

202. Brok, J., Pritchard-Jones, K., Geller, J. I. \& Spreafico, F. Review of phase I and II trials for Wilms' tumour-can we optimise the search for novel agents? Eur. J. Cancer 79, 205-213 (2017)

203. Nomura, M. et al. Tegavivint and the $\beta$-catenin/ALDH axis in chemotherapy-resistant and metastatic osteosarcoma. J. Natl Cancer Inst. 111, 1216-1227 (2019).

204. Drost, J. \& Clevers, H. Organoids in cancer research. Nat. Rev. Cancer 18, 407-418 (2018).

205. Rogers, H. J., Verhagen, M. V., Shelmerdine, S. C., Clark, C. A. \& Hales, P. W. An alternative approach to contrast-enhanced imaging: diffusion-weighted imaging and T1-weighted imaging identifies and quantifies necrosis in Wilms tumour. Eur. Radiol. 29, 4141-4149 (2019).

206. Brok, J. et al. The clinical impact of observer variability in lung nodule classification in children with Wilms tumour. Paedr. Blood Cancer 67, 4141-4149 (2020).

207. Miguez, A. C. K. et al. Assessment of somatic mutations in urine and plasma of Wilms tumor patients. Cancer Med. 9, 5948-5959 (2020).

208. US National Library of Medicine. ClinicalTrials.gov https://clinicaltrials.gov/ct2/show/NCT04322318? term $=$ NCT0 4322318\&draw $=2$ \&rank $=1$ (2021)

209. Fischbach, B. V., Trout, K. L., Lewis, J., Luis, C. A $\&$ Sika, M. WAGR syndrome: a clinical review of 54 cases. Pediatrics 116, 984-988 (2005).

210. Mueller, R. F. The Denys-Drash syndrome. J. Med. Genet. 6, 471-477 (1994). 
211. Brioude, F. et al. Overgrowth syndromes-clinical and molecular aspects and tumour risk. Nat. Rev. Endocrinol. 15, 299-311 (2019).

212. Birch, J. M. et al. Relative frequency and morphology of cancers in carriers of germline TP53 mutations. Oncogene 20, 4621-4628 (2001).

213. Kajii, T. et al. Cancer-prone syndrome of mosaic variegated aneuploidy and total premature chromatid separation: report of five infants. Am. J. Med. Genet. 104, 57-64 (2001).

214. Yost, S. et al. Biallelic TRIP13 mutations predispose to Wilms tumor and chromosome missegregation. Nat. Genet. 49, 1148-1151 (2017).

215. Reid, S. et al. Biallelic BRCA2 mutations are associated with multiple malignancies in childhood including familial Wilms tumour. J. Med. Genet. 42, 147-151 (2005)

216. Reid, S. et al. Biallelic mutations in PALB2 cause Fanconi anemia subtype FA-N and predispose to childhood cancer. Nat. Genet. 39, 162-164 (2007).

217. Kakinuma. A. et al. Familial primary hyperparathyroidism complicated with Wilms tumor. Intern. Med. 33, 123-126 (1994).

218. Szabo, J. et al. Hereditary hyperparathyroidism-jaw tumor syndrome: the endocrine tumor gene HRPT2 maps to chromosome 1q21-q31. Am. J. Hum. Genet. 56, 944-950 (1995)

219. Cunniff, C. et al. Health supervision for people with Bloom syndrome. Am. J. Med. Genet. Part. A 176, 1872-1881 (2018)

220. Astuti, D. et al. Germline mutations in DIS3L2 cause the Perlman syndrome of overgrowth and Wilms tumor susceptibility. Nat. Genet. 44, 277-284 (2012).

221. Carey, J. C. $\&$ Barnes, A. M. Wilms tumor and trisomy 18: is there an association? Am. J. Med. Genet. C. Semin. Med. Genet. 172, 307-308 (2016)

222. Karlberg, N. et al. High frequency of tumours in Mulibrey nanism. J. Pathol. 218, 163-171 (2009).

223. Sivunen, J. et al. Renal findings in patients with Mulibrey nanism. Pediatr. Nephrol. 32, 163-171 (2017).

224. Perotti, D. et al. Is Wilms tumor a candidate neoplasia for treatment with WNT/ $\beta$-catenin pathway modulators?-A report from the Renal Tumors BiologyDriven Drug Development Workshop. Mol. Cancer Ther. 12, 2619-2627 (2013).

225. Wolpaw, A. J. et al. Drugging the 'undruggable' MYCN oncogenic transcription factor: overcoming previous obstacles to impact childhood cancers. Cancer Res. 81, 1627-1632 (2021)

226. Maschietto, M. et al. The IGF signalling pathway in Wilms tumours-a report from the ENCCA Renal Tumours Biology-Driven Drug Development Workshop. Oncotarget 5, 8014-8026 (2014).

227. Meadows, A. T. et al. Patterns of second malignant neoplasms in children. Cancer 40, 1903-1911 (1977).

228. Lemerle, J. et al. Preoperative versus postoperative radiotherapy, single versus multiple courses of actinomycin D, in the treatment of Wilms' tumor. Preliminary results of a controlled clinical trial conducted by the International Society of Paediatric Oncology (S.I.O.P.). Cancer 38, 647-654 (1976).

229. Graf, N. et al. Fifty years of clinical and research studies for childhood renal tumors within the International Society of Pediatric Oncology (SIOP). Ann. Oncol. https://doi.org/10.1016/j.annonc.2021. 08.1749 (2021).

230. Tournade, M. F. et al. Results of the Sixth International Society of Pediatric Oncology Wilms' tumor trial and study: a risk-adapted therapeutic approach in Wilms' tumor. J. Clin. Oncol. 11, 1014-1023 (1993).

231. de Kraker, J. et al. Wilm's tumor with pulmonary metastases at diagnosis: the significance of primary chemotherapy. International Society of Pediatric Oncology Nephroblastoma Trial and Study Committee. J. Clin. Oncol. 8, 1187-1190 (1990).

232. Green, D. M. et al. Comparison between single-dose and divided-dose administration of dactinomycin and doxorubicin for patients with Wilms' tumor: a report from the National Wilms' Tumor Study Group. J. Clin. Oncol. 16, 237-245 (1998).

233. De Camargo, B. \& Franco, E. L. A randomized clinical trial of single-dose versus fractionated-dose dactinomycin in the treatment of Wilms' tumor. Results after extended follow-up. Cancer 73 , 3081-3086 (1994).

234. International Agency for Research on Cancer. Estimated age-standardized mortality rates (world) in 2020, kidney, both sexes, ages 0-14. IARC https:// gco.iarc. fr/today/online-analysis-map? $\mathrm{v}=2020 \AA$ mode=population\&mode_population=continents $\delta$ population $=900 \&$ populations $=900 \&$ key $=$ asr $\&$ sex $=$ $0 \&$ cancer $=29 \&$ type $=1 \&$ statistic $=5 \&$ prevalence $=$ $0 \&$ population group $=0$ \&ages group $\% 5 \mathrm{~B} \% 5 \mathrm{D}=0 \AA$ ages_group $\% 5 \mathrm{~B} \% 5 \mathrm{D}=2 \& \mathrm{nb}$ _items=10\&group_ cancer $=1$ \&include_nmsc $=1$ \&include_nmsc_other= 1 \&projection=natural-earth \&color palette $=$ default\&map_scale=quantiledmap_nb_colors $=5 \bar{\alpha}$ continent $=0 \&$ show_ranking $=0$ \&rotate $=\% 255 \mathrm{~B} 10 \%$ 252C0\%255D (2020).

235. Johnson, B. K. \& Comstock, R. D. Epidemiology of chest, rib, thoracic spine, and abdomen injuries among United States high school athletes, 2005/06 to 2013/14. Clin. J. Sport. Med. 27, 388-393 (2017).

236. Kim, J. K. et al. A systematic review of genitourinary injuries arising from rugby and football. J. Pediatr. Urol. 16, 130-148 (2020).

\section{Acknowledgements}

The authors thank both the SIOP Renal Tumour Study Group and the Children's Oncology Group Renal Tumour Committee for their collective expertise. Their work laid the foundations for this Review article. The authors also thank parents and survivors of childhood Wilms tumour for their contribution to setting research priorities.

\section{Author contributions}

Introduction (F.S. and K.P.-J.); Epidemiology (F.S., K.P.-J. and K.N.); Mechanisms/pathophysiology (F.S., K.P.-J., M.G., M.M. and S.B.); Diagnosis, screening and prevention (F.S., K.P-J., C.V.F., J.B., S.L.-F. and G.V.); Management (F.S., C.V.F., K.P.-J, S.L.-F. and V.P.); Quality of life (F.S., K.P.-J. and A.P.); Outlook (F.S., K.P.-J., C.V.F., J.B., M.G., S.L.-F. and J.I.G.); Overview of the Primer (F.S. and K.P.-J.)

\section{Competing interests}

All authors declare no competing interests.

Peer reviewer information

Nature Reviews Disease Primers thanks R. Furtwängler, $\mathrm{N}$. Cost, J. Kalapurakal and the other anonymous reviewer(s) for their contribution to the peer review of this work.

\section{Publisher's note}

Springer Nature remains neutral with regard to jurisdictional claims in published maps and institutional affiliations.

\section{Supplementary information}

The online version contains supplementary material available at https://doi.org/10.1038/s41572-021-00308-8.

(c) Springer Nature Limited 2021 